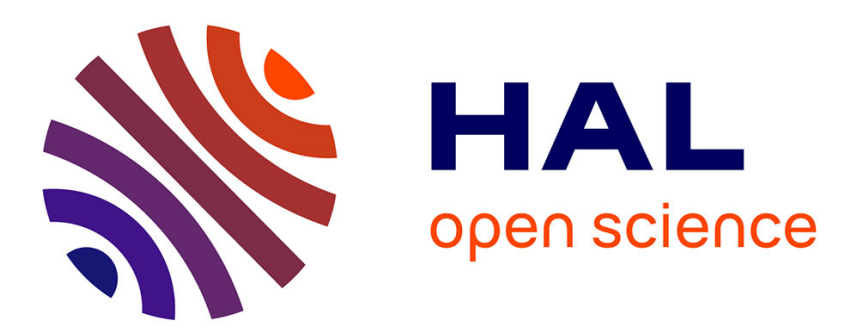

\title{
Mapping of quadrature magnetic susceptibility/magnetic viscosity of soils by using multi-frequency EMI
}

François-Xavier Simon, Apostolos Sarris, Julien Thiesson, Alain Tabbagh

\section{To cite this version:}

François-Xavier Simon, Apostolos Sarris, Julien Thiesson, Alain Tabbagh. Mapping of quadrature magnetic susceptibility/magnetic viscosity of soils by using multi-frequency EMI. Journal of Applied Geophysics, 2015, 120, pp.36-47. 10.1016/j.jappgeo.2015.06.007 . hal-01376254

\section{HAL Id: hal-01376254 https://hal.sorbonne-universite.fr/hal-01376254}

Submitted on 4 Oct 2016

HAL is a multi-disciplinary open access archive for the deposit and dissemination of scientific research documents, whether they are published or not. The documents may come from teaching and research institutions in France or abroad, or from public or private research centers.
L'archive ouverte pluridisciplinaire HAL, est destinée au dépôt et à la diffusion de documents scientifiques de niveau recherche, publiés ou non, émanant des établissements d'enseignement et de recherche français ou étrangers, des laboratoires publics ou privés. 
1 Mapping of quadrature magnetic susceptibility/magnetic viscosity of soils by using multi-frequency EMI

3

4 François-Xavier Simon ${ }^{1}$, Apostolos Sarris ${ }^{1}$, Julien Thiesson ${ }^{2}$, Alain Tabbagh ${ }^{2}$

${ }^{1}$ Lab GeoSat ReSeArch, IMS-FORTH, Rethymno

${ }^{2}$ Sorbonne Universités, UPMC -Paris6, UMR7619-Métis, F-75252, Paris magnetic susceptibility about the history of soils. In the field its mapping can be achieved by TDEM measurement. Here we study the applicability of multi-frequency FDEM viscosity measurement in the low frequency range using a commercial EMI instrument. The dependence of the in-phase and quadrature out-of-phase components of the ratio of secondary magnetic field to primary magnetic field, electrical conductivity, magnetic susceptibility and magnetic viscosity is first described. The procedure allowing the determination of the three apparent properties is then proposed. It delivers first the conductivity using the differences between the quadrature responses at two different frequencies. Then, after removing the conductivity effects both in the in-phase and quadrature components, it provides the values of the magnetic susceptibility and viscosity. This procedure is tested on $1 \mathrm{D}$ and 3D synthetic cases to assess any arising uncertainty. The application of the method is attested in two archaeological case histories in Thessaly in conductive and magnetic soil contexts. The apparent magnetic viscosity maps are significantly different from magnetic susceptibility and conductivity maps thus bringing new information into the game. 


\section{Keywords}

Low induction number, EMI, soil magnetic viscosity, soil magnetic susceptibility, soil electrical conductivity, multi-frequency.

\section{Introduction}

Frequency domain electromagnetic induction (EMI) instrument with Slingram geometry (dipole-dipole) is of common use in archaeological prospection (Scollar et al., 1990) and soil studies (Corwin and Lesch, 2005). It allows mapping simultaneously electrical conductivity and magnetic susceptibility of soils. These light mobile instruments present also the advantage to be easily towed on the field (De Smedt et al., 2013).

For the low frequency domain, the choice to neglect displacement currents limits the used frequencies to $100 \mathrm{kHz}$ and the geometric scale of the instruments, i.e. the metric intercoil separation, determines the investigated volume. Consequently these EMI instruments respect the low induction number (LIN) approximation and thus: (1) the conductivity response is in quadrature out-of-phase from the transmitter moment that allows the measurement of the in-phase magnetic susceptibility, (2) the depth of investigation is only governed by the instrument's geometry. Only geometrical soundings can be achieved; therefore there is no advantage in using different frequencies.

Until now, with EMI frequency domain instruments, only the in-phase magnetic susceptibility is measured, while quadrature susceptibility/magnetic viscosity has been measured and mapped using TDEM instruments (Thiesson et al. 2007). However, some observations of this property with single frequency instruments have been also achieved (Mc Neill 2013).

To improve the interpretation of FDEM measurements one faces a series of difficulties; on one hand the magnetic susceptibility is a complex quantity which generates a 
quadrature response that algebraically adds to the conductivity response and, on the other hand, the LIN approximation and low frequency approximation have their own limits. When either the conductivity or the frequency increases, a significant in-phase response appears that algebraically adds to the in-phase susceptibility response. Over clayed soils, the dielectric permittivity can also be sufficiently high to generate measurable responses in the higher portion of the low frequency range.

The use of several frequencies has been considered to overcome these limitations. Limiting the purpose of the present paper to magnetic properties, it is first preferable to stay in the lower part of the frequency range around or below $10 \mathrm{kHz}$. In this range, due to the absence of frequency dependence of the quadrature component of soil magnetic susceptibility (Mullins and Tite 1973), it is possible to separate its response from the one generated by the conductivity, which increases with frequency. This solution has already been proposed (Tabbagh, 1986a) and a two-frequency prototype instrumentation has been tested (Benech, 2000).

Since 1996, a multi-frequency commercial instrument, the GEM-2, has become available. Its manufacturers claimed that it allows frequency soundings (Won et al., 1996). This statement raised a contestation (Mc Neill, 1996) recalling the LIN approximation implications. The argument was closed by the admission (Huang and Won, 2003) that frequency soundings can only be relevant for higher induction number conditions when the ground conductivity is sufficiently high (sea water bathymetry). Nevertheless, it remains interesting to check the results that can be obtained in practice with such multifrequency instrument for both electrical conductivity and complex magnetic susceptibility. In particular, the TDEM measurements, used to map of the quadrature susceptibility/magnetic viscosity, indicated spatial variations that can be significantly different from those of the magnetic 
$96 \frac{2}{\pi} \kappa_{q u}=-\frac{\partial \kappa_{p h}(\omega)}{\partial \operatorname{Ln}(\omega)}$ (Thiesson et al., 2012). mapping.

susceptibility and can be more clearly linked with nitrogen or carbon content of a soil

In the present paper, after a short recall about the soil electromagnetic properties and the theoretical responses they generate, we will consider synthetic and experimental results obtained in different sites and discuss about the reliability of quadrature susceptibility

\section{Measured soil properties}

In the frequency range where the displacement currents proved to be neglected (below $100 \mathrm{kHz}$ ), the electrical resistivity, $\rho$, is well defined and extends from 1 to $2 \Omega . \mathrm{m}$ in the intertidal zone to $10000 \Omega$.m in permafrost or in crystalline dry soils. This very wide dynamic range is the greatest observed for usual geophysical properties, but values out of the $[10,1000$ $\Omega . m]$ interval remain occasional in soils studies. In accordance with the hypothesis where the polarization effects are negligible, in the particular range the conductivity is assumed independent of the frequency.

On the contrary, the magnetic susceptibility is complex and varies with frequency (Mullins, 1977); it must thus be written: $\kappa(\omega)=\kappa_{p h}(\omega)-i \kappa_{q u}(\omega)$ where $\omega$ is the angular frequency, $\kappa_{p h}$ is the in-phase component and $\kappa_{q u}$ the quadrature component. However, all the experiments, except those from iron working sites, undertaken over laboratory samples respect the dispersed single-domain grain theory (Néel, 1949) which states that the quadrature component is constant and linked to the frequency variation of the in-phase part by:: 
(Mullins and Tite 1973, Dabas et al. 1992, Dabas and Skinner 1993). This decrease of the inphase susceptibility and the value of the quadrature susceptibility correspond to the same parameter called magnetic viscosity. The viscosity is also measured in the field using TDEM instruments (Colani and Aitken 1966, Thiesson et al. 2007) and all the field results, (see for example Pétronille et al. 2010), correspond to a slope of the logarithmic decrease close to -1 for the $\frac{\partial B(t)}{\partial t}$ signal measured in the receiver coil in accordance with the above formula. The in-phase soil susceptibility range of values is also quite large, from $100010^{-5} \mathrm{SI}$ in volcanic areas to $1010^{-5} \mathrm{SI}$, but values close to $100010^{-5} \mathrm{SI}$ are rare. Due to the presence of small mono-domain/superparamagnetic grains the quadrature susceptibility is significant and more often of the order of $6 \%$ of the in-phase susceptibility.

Consequently we will interpret the soil responses to a low frequency field excitation by considering a constant conductivity and a susceptibility following equation (1). The calculation of the response of a homogeneous ground, or of layered one, has been established by the work of Wait (1959), and is based on Hankel's transforms that can be rapidly calculated using convolution products (Guptasarma and Singh, 1997). Complete expressions and a description of the different subsequent approximations can be found in (Thiesson et al. 2014). They allow expressing the measurement in terms of apparent conductivity and apparent susceptibility, based on a simplified conversion since the variations are monotonous although not simply linear.

To simplify the expressions in the following parts of the text, one will use the term magnetic susceptibility for the in-phase part only and use the term magnetic viscosity for the quadrature out-of-phase part. 


\section{GEM-2 instrument specificity}

In this study we process data acquired with the GEM-2 instrument (Figure 1). This multi-frequency instrument is not a simple coplanar coil configuration Slingram device, but instead it has in fact two receivers coils, one serving as bucking coil for the other (Won et al. 1996). They have the same surface and are mounted in opposite directions in such way that the primary field is exactly compensated: the "bucking" coil at a $1.035 \mathrm{~m}$ distance from the transmitter coil having four times less turns than the "receiver" located at $1.66 \mathrm{~m}$ distance. The measured quantity is thus: $\frac{\left(H_{s R}-H_{s B} / 4\right)}{H_{p}}$, where $H_{p}$ is being the primary field at the 'receiver' location, $H_{s R}$ the secondary field at the 'receiver' location and $H_{s B}$ the secondary field at the 'bucking' location. Thus, one must first reconsider the relationships between this measured quantity and the ground properties.

Figure 2 presents the responses for both conductivity and in-phase susceptibility (the quadrature susceptibility being $6 \%$ of the in-phase) at $5 \mathrm{kHz}$ and $40 \mathrm{kHz}$ for the characteristics of the GEM-2 instrument when held at $0.3 \mathrm{~m}$ height above the ground surface. It can be observed that while the quadrature response is simply correlated (quasi proportional) with the ground conductivity, the in-phase sensitivity to susceptibility changes is hampered by the inphase conductivity response which seems totally dominating the response for lower susceptibilities at $40 \mathrm{kHz}$.

Another important aspect is the dependence on the height above the ground, as the HCP configuration is known to exhibit changes from positive to negative values in the inphase response when the altitude increases (Tabbagh, 1986a). Figure 3 shows that while the quadrature response monotonically decreases with the altitude $\mathrm{H}$, the in-phase one exhibits a sharp maximum around $\mathrm{H}=0.3 \mathrm{~m}$. This response is shifted towards negative values when the 
conductivity increases but it retains the same shape. This effect of the HCP configuration considerably affects the shape and the sign of the anomaly for the apparent magnetic susceptibility as its vertical distribution varies due to the pedogenesis process and sedimentary disturbance.

As can be observed in Figure 4 the response generated by the quadrature susceptibility is linear but of quite limited magnitude compared to the electrical conductivity response. This effect explains the invisibility of the magnetic viscosity on the raw data which more often appear only related to the electrical conductivity.

\section{Methodology}

The aim of any type of geophysical prospection is to identify the physical properties and the geometrical shapes of the different media present in the underground. This is achieved through a full inversion process but before implementing this heavy process, and to be able to start it with a relevant guess of a priori parameter values, it is necessary to have a first assessment of the information by transforming the raw data to apparent property variations. The general definition of an apparent property is the one of a homogeneous ground that would deliver the same measurement with the same instrument. Establishing apparent electrical conductivity, susceptibility and viscosity maps is the purpose of the present work but as several properties intervene the task is more difficult than when only one property can be considered, as is the case in D.C. resistivity prospecting where the transformation corresponds to a simple multiplication by a coefficient. Thus, it is necessary to describe the successive steps of the transformation of the raw data in apparent properties because different procedures would lead to (slightly but certainly) different results. 

into experimental $(\mathrm{Hs} / \mathrm{Hp})$ ratio in $\mathrm{ppm}$, established on a methodology described by Thiesson et al. 2014). For the quadrature channel it is based on a comparison between an electrical sounding and measurements at (at least) two heights above the ground surface. In this case, we assume that the higher measurement is almost not affected by the magnetic properties but only by electrical conductivity. Thus, one obtains the calibration coefficient between the digits measured by the instrument and the theoretical response for the quadrature part of the EM signal. In the second calibration step the experimental in-phase response acquired with an aluminum sphere is compared with the theoretical one (Thiesson et al. 2014). For our instrument, in-phase coefficient is -0.70 digit $/ \mathrm{ppm}$ and the quadrature coefficient -1.0 digit/ppm. We repeat this calibration at each different field of survey, to ensure the stability of the instrument.

To extract apparent complex magnetic susceptibility it's required to beforehand calculate the electrical conductivity. Multi-frequency measurements allow removing the effect of the magnetic viscosity on the quadrature part of the signal. Considering the difference between two different frequencies this difference is then transformed in apparent electrical conductivity by correspondence with a reference curve calculated using the complete formulas (Thiesson et al. 2014).

In the second step of the procedure, the conductivity value is used to calculate the inphase and quadrature parts of the signal generated by the electrical conductivity at each frequency. By subtracting these parts from the experimental data one can remove the conductivity parts on both in-phase and quadrature channels and retain the parts generated by the susceptibility and the viscosity. Magnetic viscosity strictly affects quadrature out-of-phase part of the EM signal while the magnetic susceptibility only affects the in-phase part of the signal. These imaginary and real parts of the secondary to primary field ratio, in ppm, are then 
expressed in the apparent properties by comparing the values with master curves (magnetic susceptibility as a function of in-phase part of the EM signal in ppm, and magnetic viscosity as a function of quadrature out-of-phase part of the EM signal in ppm). The master curves were beforehand calculated using the full analytical solution.

\section{Synthetic data}

The advantages of synthetic data processing is to evidence the difficulties that can exist in the process before any perturbations generated during field work, the introduction of external noise and possible instrument default or failure(s). Synthetic data will be analyzed through a $1 \mathrm{D}$ case and a simple 3D geometry case.

\section{$1 D$ synthetic data}

As the raw data transformation applied to homogeneous ground responses restitutes the exact values of the three properties one considers here a two layers model. The first layer $\left(\rho=100 \Omega . \mathrm{m}, \kappa_{\text {phf } 1}=100.10^{-5} \mathrm{SI}, \kappa_{\mathrm{phf} 2}=95.6 .10^{-5} \mathrm{SI}, \kappa_{\mathrm{qu}}=6.10^{-5} \mathrm{SI}\right)$ has an increasing thickness, starting to 0.1 meter up to 3 meter, the second one is a more conductive and less magnetic $\left(\rho=50 \Omega . \mathrm{m}, \kappa_{\mathrm{phf} 1}=10.10^{-5} \mathrm{SI}, \kappa_{\mathrm{phf} 2}=9.56 .10^{-5} \mathrm{SI}, \kappa_{\mathrm{qu}}=0.6 .10^{-5} \mathrm{SI}\right)$. The susceptibility variation with frequency respects equation (1) for the frequencies of 5010 and $13370 \mathrm{~Hz}$ used by GEM2. We simulated the response for the GEM-2 in a HCP configuration, at $0.3 \mathrm{~m}$ elevation. The variation of the apparent susceptibility is shown in Figure 5a and that of the apparent viscosity in Figure 5b. We observe a slight influence of the frequency that increases with the thickness of the first layer; it is low but clearly observable for the viscosity due to the small values of it. This corresponds to the apparent conductivity change, even if not totally corrected in the two layer case by the applied procedure: the slope of the quadrature response against viscosity is slightly dependent on the conductivity. The apparent magnetic viscosity delivered by the used 
procedure is thus in (small) dependence on the electrical conductivity as shown in Figure 6 for both 5010 and 13370 frequencies. In this Figure the absence of conductivity dependence would correspond to horizontal straight lines for each magnetic viscosity value, and globally one can observe that its dependence is low. However, one must notice that the discrepancy (1) increases with frequency, (2) is, as expected, high for smaller viscosity values, but (3) increases also for high resistivity. In this last case the uncertainty is generated by the uncertainty in the evaluation of the resistivity itself.

Finally this example underlines that the determination of an apparent magnetic viscosity value using the proposed procedure is still valuable for medium and high magnetic viscosity (still as a possible value for common soil). As the discrepancy is frequency dependent, the determination of the magnetic viscosity with the lowest frequencies is much more preferable.

\section{$3 D$ synthetic data}

3D synthetic data were simulated by the moment method (Tabbagh, 1985). We put a resistive and magnetic block $\left(\rho=500 \Omega \mathrm{m}, \kappa_{\mathrm{phf} 1}=150.10^{-5} \mathrm{SI}, \kappa_{\mathrm{phf} 2}=143.10^{-5} \mathrm{SI}, \kappa_{\mathrm{qu}}=10.10^{-5} \mathrm{SI}\right)$ in a two layers medium. The first layer, of $0.2 \mathrm{~m}$ thickness, is more resistive and magnetic $\left(\rho=100 \Omega \mathrm{m}, \kappa_{\text {phf1 }}=50.10^{-5} \mathrm{SI}, \kappa_{\mathrm{phf} 2}=49.10^{-5} \mathrm{SI}, \kappa_{\mathrm{qu}}=3.10^{-5} \mathrm{SI}\right)$ than the second one $(\rho=50 \Omega \mathrm{m}$, $\left.\kappa_{\mathrm{phfl}}=10.10^{-5} \mathrm{SI}, \kappa_{\mathrm{phf} 1}=9.10^{-5} \mathrm{SI}, \kappa_{\mathrm{qu}}=1.10^{-5} \mathrm{SI}\right)$ as we have usually noticed on well-drained soil. In accordance with the Neel's theory the in-phase magnetic susceptibility still decreases with the frequency, depending on the value of the magnetic viscosity. The target is a block of $2 \times 2 \times 2 \mathrm{~m}^{3}$ centered at a depth of $1.2 \mathrm{~m}$, i.e. entirely in the second layer. The top of this target is close to the ground surface. This simulation uses the same acquisition parameters as in-field acquisition: a 1m measurement step, an altitude of 0.3 meter in the HCP configuration and with the same instrumental settings. We again used the two first frequencies of the 
instrument, $5010 \mathrm{~Hz}$ and $13370 \mathrm{~Hz}$. The synthetic data are then processed with the previously introduced procedure. We obtain 5 maps (Fig. 7): electrical conductivity on the one hand, magnetic susceptibility and magnetic viscosity at both frequencies on the other hand.

As expected from previous theoretical studies (Tabbagh 1986b) for HCP configuration, the apparent electrical conductivity shows a complex shaped anomaly, which even for such a simple shaped target has three extrema. The shape of the apparent magnetic susceptibility contours is simpler than the electrical one. It shows a high value just above the center of the target and weak negative values around the block, but the shape of the anomaly and the shape of the target present differences. This point needs to be taken into account in the further interpretation steps. Considering the frequencies, both maps are exactly similar. For both frequencies the modes of apparent in-phase (susceptibility) and quadrature (viscosity) values are governed by the instrument geometry and without link with the anomalous causative body. We thus consider the variation of the data expressed by the difference between the maximum and minimum values of the anomaly on each map. At $5010 \mathrm{~Hz}$ frequency the differences are $20410^{-5} \mathrm{SI}$ in-phase and $15.310^{-5} \mathrm{SI}$ in quadrature. This corresponds to a ratio of $7.5 \%$ between the two components. At $13370 \mathrm{~Hz}$ frequency the corresponding values are $184.910^{-5}$ SI, $15.510^{-5}$ SI and $8.4 \%$. The amplitude of the in-phase susceptibility slightly decreases with frequency while the quadrature (viscosity) practically remains constant (considering the error resulting from the non-strictly addition of magnetic viscosity and electrical conductivity) in agreement with the susceptibility values introduced in the modelling. The viscosity/susceptibility ratio is slightly higher than the ratio of the body properties $(6.7 \%)$ but this can also be explained by the influence of the higher ratio chosen for the second layer $(11.1 \%)$. 
We employed the GEM-2 in two archaeological sites to map the complex magnetic susceptibility and the electrical conductivity. These two sites are located on the Thessaly plain in central Greece. The first one, Karatzantakli, in the mountain area, is covered by a high clay content soil which suggests a high conductivity. The second one, Almiriotiki, in plain, close to paleo-channel and floodplain deposits has also high clay content but with strong heterogeneities affecting the electrical conductivity. These human settlements are likely to change magnetic properties of soils and generate anomalies related to the archaeological features and handcraft activities area. It also looks well-suited to our studies. In-phase parts of the signal are affected by the electrical conductivity. As showed on the methodological part, the use of different frequencies allows removing its first order in-phase and quadrature effects. We are thus able to map the magnetic viscosity distribution over the area cover by the EM data and observe its significance.

Five different frequencies from $5010 \mathrm{~Hz}$ until 40050Hz $(5010 \mathrm{~Hz}, 13370 \mathrm{~Hz}, 22530$ $\mathrm{Hz} 31290 \mathrm{~Hz}$ and $40050 \mathrm{~Hz}$ ) were used. Measurements were continuously acquired almost every meter along profiles $1 \mathrm{~m}$ apart. The instrument was carried at an altitude of $0.3 \mathrm{~m}$ above the ground surface. Despite manufacturer advice, we preferred to carry the instrument at this height regarding the curve of sensitivity for the magnetic properties on the in-phase part of the instrument. Only the two lower frequencies are considered here for magnetic properties mapping.

\section{Karatzantakli}

Karatzantakli is a Neolithic tell ("magoula"), consisting of an accumulation of anthropogenic material, which means high soil heterogeneity. On this site geomorphological variations are induced by different cover material in the different parts of the site. Some 
features like kilns or fireplaces could increase considerably the magnetic susceptibility or the magnetic viscosity at some points. Measurements were recorded in a manual mode. Measurements were automatically acquired along the profile with a regular distribution of the measurements along each one.

The raw data, in Figure 8, show high contrast on the quadrature part of the signal and on the in-phase part of the EM signal. For the in-phase part of the signal, ranges of values are similar at both frequency but with a marked offset. On the quadrature out-of-phase part of the signal, the range of values for the higher frequency is approximately two times higher than for the lower frequency and both show a high conductivity who do affect the in-phase part of the EM signal (Fig. 8).

The five maps resulting from the raw data processing are presented in Figure 9. Apparent electrical conductivity shows some differences between the left and right parts of the area.. The important depth of investigation for this instrument in HCP geometry doesn't allow any shallow characterization of the soils and it is more representative of a too thick layer of soil.Apparent magnetic susceptibility shows more anomalies on the whole area. Values of susceptibility are extremely high. For $5010 \mathrm{~Hz}$ the median of values is $227.10^{-5} \mathrm{SI}$ and the interquartile is $73.10^{-5}$. These values of magnetic susceptibility correspond with very high values of magnetic viscosity due to the inter dependence of magnetic susceptibility and magnetic viscosity. The inter-quartile difference changes from $73.10^{-5}$ SI to $71.10^{-5}$ between the two frequencies. Decreasing of interquartile seems very small regarding the high value of magnetic susceptibility but could be also an effect of the distribution of the magnetic susceptibility. Some anomalies are common on both magnetic susceptibility and magnetic viscosity but generally speaking the maps look quite different. This means that the ratio of magnetic viscosity on magnetic susceptibility is varying from on part to another one which implies different compositions regarding the size of the magnetic grains. These differences 
confirm the interest to be attentive to this soil property regarding the EM signal. The range of values of the ratio between magnetic viscosity interquartile value and magnetic susceptibility interquartile value is close to $26 \%$. This high value could probably be explained by an insufficiently good relative gain calibration between the in-phase and quadrature channels.

\section{Almiriotiki}

The second field test is the Neolithic tell of Almiriotiki. Measurements were acquired with a GPS RTK (Javad Triumph) which allows covering a very large area in one day (around 2 ha/day). Accuracy of the positioning is better than decimetric due to the differential correction of the GPS point. This site presents a settlement covering the top part of the tell (magoules) and a bottom part around the central elevation.

We present the raw data for the in-phase part and the quadrature parts of the EM signal in Figure 10. For the in-phase part we observe an offset between the two frequencies but the same dynamic range of values. Only small differences can be attributed to the electrical conductivity, especially in the north part of the map. The quadrature part of the signal shows two similar maps for the both frequencies, with contrasted ranges of values. This frequency evolution is the consequence of the dependence on the frequency of the electrical conductivity measurements. Both frequency and dynamic range of values are approximately multiplied by a factor of two. At first glance the part of magnetic properties is invisible on both quadrature and in-phase response maps.

The processing allows us to obtain the five different maps presented in Figure 11. In appearance, electrical conductivity is very close to the raw data due to the strong effect of the electrical conductivity on the quadrature part of the signal. The values of the conductivity show a global high conductivity. On the north part, the conductivity increases, probably due to the soil modification induced by flooding deposits. It explains the effect of the conductivity 
on the in-phase part of the raw data for the same location. The tell is more resistive due to the mix of anthropogenic material and natural clay soils. For the susceptibility, interpretation is less obvious which is not surprising regarding the HCP configuration.

Both magnetic viscosity maps are very similar. In this case data are noisier than in previous example. Nevertheless value of magnetic susceptibility between 100 and $300.10^{-5}$ S.I. is still very high. Regarding the link between the magnetic susceptibility and the magnetic viscosity we can again expected high value of magnetic viscosity. Ratio of magnetic susceptibility and magnetic viscosity (inter-quartiles) is still higher than expected and close to $22 \%$. Again, this effect could be derived from a poor calibration but again the viscosity maps, very coherent between the two frequencies, show underground patterns significantly different from those shown by susceptibility maps. This again demonstrates the high interest of viscosity measurements.

\section{Discussion}

The experiments here presented were acquired using the GEM-2 instrument in HCP configuration. This configuration and the existence of two receivers do not facilitated the interpretation but this manufacturer's design implies a better stability when moving the instrument on the field. In near future, it is necessary to implement the use of VCP geometry by improving the stability of the instrument and correcting for the slight modification of the axis orientation during a continuous acquisition. Regarding the greater simplicity of VCP sensitivity curves, these improvements will allow a better description of the magnetic viscosity underground variations. However, in its present configuration the GEM-2 is already usable to perform a significant and qualitatively reliable magnetic viscosity mapping at the same time as conductivity and magnetic susceptibility mappings. 

raw data in apparent properties is not straightforward but it is efficient. If the quantitative

362

363 interpretation must use the raw data, the mapping of apparent properties proves to be feasible and informative. As the way to express the apparent properties is clearly established and the uncertainty assessed, it is possible to take into account the potential error on the estimation. The adopted definition of the apparent properties is thus satisfactory; especially because the error effect of this assumption is lower than the spatial variability of soil properties. Nevertheless, this error adds to the possible different gains between the in-phase and quadrature measurement channels, and prevents any precise quantitative determination of the ratio between the magnetic susceptibility and the magnetic viscosity, which can open the way to study the in-field variations of the grain size distributions. In all published magnetic viscosity measurements in the field (Thiesson et al. 2007, Pétronille et al. 2010), the maps of this ratio were informative and from the archaeological point of view supported assumptions about the functions of the detected features (metallurgy, pottery, domestic waste). In paleosoil studies too, when this ratio is recalculated from the frequency dependence of the in-phase magnetic susceptibility, this ratio is more discriminant for layer identification than the susceptibility itself (Thiesson 2007)

\section{Conclusion}

The first series of tests and experiments aiming at mapping the magnetic viscosity, together with the electrical conductivity and the magnetic susceptibility, using a commercial multi-frequency FDEM instrument have been demonstrated. In spite that the instrument characteristics and its coil configuration are far from optimal for soil studies, the results are convincing and the experiments confirm its effectiveness in mapping this parameter: the 
viscosity maps are not simple traces of the in-phase component of the magnetic susceptibility. Further comparisons with TDEM measurements are planned to elucidate the relative advantages of both ways of measurement. For FDEM, going ahead in the study of the magnetic grain sizes distribution necessitates to adopt a more convenient coil configuration and to progress in the relative gain calibration between in-phase and out-of-phase channels.

In the two examples examined, only the two lower frequencies were taken into account. For these frequencies the proportionality of the quadrature response with the conductivity is almost exact and only in high conductivity areas the effect on the in-phase part of the signal can be observed. When increasing the frequency, another parameter may affect the responses, namely the dielectric permittivity (Huang and Fraser, 2001). A significant amount of work must yet to be undertaken to assess all the possibilities offered by low frequency EMI instruments in soil study contexts.

\section{Acknowledgments}

This work was performed in the framework of the IGEAN ("Innovative Geophysical Approaches for the study of Early Agricultural villages of Neolithic") project which is implemented under the "ARISTEIA" Action of the "OPERATIONAL PROGRAMME EDUCATION AND LIFELONG LEARNING" and is co-funded by the European Social Fund (ESF) and National Resources. 


\section{References}

Benech, C., 2000. Interprétation conjointe de cartographies magnétique et électromagnétique des propriétés magnétiques des sols anthropisés. UPMC, Paris.

Colani, C., Aitken, M.J., 1966. A new type of locating device. I field trials. Archaeometry, 9, 9-19.

Corwin, D.L., Lesch, S.M., 2005. Characterizing soil spatial variability with apparent soil electrical conductivity: I. survey protocols. Computers and Electronics in Agriculture, 46, 103-133.

Dabas, M., Jolivet, A., Tabbagh, A., 1992. Magnetic susceptibility and viscosity of soils in a weak time varying field. Geophysical Journal International, 108, 101-109.

Dabas, M., Skinner, J., 1993. Time-domain magnetization of soils (VRM), experimental relationship to quadrature susceptibility. Geophysics, 58, 326-333.

De Smedt, P., Saey, T., Lehouck, A., Stichelbaut, B., Meerschman, E., Islam, M.M., van DeVijver, E., van Meirvenne, M., 2013. Exploring the potential of multi-receiver EMI survey for geoarchaeological prospection: A 90 ha dataset. Geoderma, 199, 30-36.

Guptasarma, D., Singh, B., 1997. New digital linear filters for Hankel J0 and J1 transforms. Geophysical Prospecting, 45, 745-762.

Huang, H., Fraser, D., 2001. Mapping of the resistivity, susceptibility, and permittivity of the earth using a helicopter-borne electromagnetic system. Geophysics, 66, 148-157.

Huang, H., Won, I., 2003. Real-time resistivity sounding using a hand-held broadband electromagnetic sensor. Geophysics, 68, 1224-1231.

Mc Neill, J.D., 1996. Why doesn't Geonics limited build a multi-frequency EM31 or EM38? Technical note TN-30, Geonics 1td, 5p.

Mc Neill, 2013. The magnetic susceptibility of soils is definitely complex. Technical note TN36, Geonics 1td, 27p. 
Mullins, C.E., 1977. Magnetic susceptibility of the soil and its significance in soil science - a review. Journal of. Soil Science, 28, 223-246.

Mullins, C.E., Tite, M.S., 1973. Magnetic viscosity, quadrature susceptibility, and frequency dependence of susceptibility in single-domain assemblies of magnetite and maghemite. Journal of. Geophysical. Research. 78, 804-809.

Néel, L., 1949. Théorie du traînage magnétique des ferromagnétiques en grains fins avec application aux terres cuites. Annales de Géophysique, 5, 99-136.

Pétronille, M., Thiesson, J., Simon, F.-X., Buchsenschutz, O., 2010. Magnetic signal prospecting using multiparameter measurements: the case study of the Gallic Site of Levroux. Archaeological. Prospection, 17, 141-150.

Scollar, I., Tabbagh, A., Herzog, I., Hesse, A., 1990. Archaeological prospecting and remote sensing, Cambridge University Press.

Tabbagh, A., 1985. The response of a three-dimensional magnetic and conductive body in shallow depth electromagnetic prospecting. Geophysical Journal of the Royal Astronomical Society 81, 215-230.

Tabbagh, A., 1986a. Applications and advantages of the Slingram electromagnetic method for archaeological prospecting. Geophysics, 51, 576-584.

Tabbagh, A., 1986b. What is the best coil orientation in the slingram electromagnetic prospecting method?, Archaeometry, 28, 185-196.

Thiesson, J., Boulonne, L., Buvat, S., Jolivet, C., Ortolland, B., Saby, N., 2012. Magnetic properties of the French soil monitoring network: first results. Near Surface Geoscience 2012 - 18th European Meeting of Environmental and Engineering Geophysics, EAGE. 
Thiesson, J., Kessouri, P., Schamper, C., Tabbagh, A., 2014. Calibration of frequency-domain electromagnetic devices used in near-surface surveying. Near Surface Geophysics, 12, 481-491.

Thiesson J; 2007. Mesure et cartographie de la viscosité magnétique des sols. PhD thesis, UPMC university, Parirs.

Thiesson, J., Tabbagh, A., Flageul, S., 2007. TDEM magnetic viscosity prospecting using a Slingram coil configuration. Near Surface Geophysics, 5, 363-374.

Wait, J., 1959. Induction by an oscillating magnetic dipole over a two layer ground. Applied. Science Research, B 7, 73-80.

Won, I., Keiswetter, D., Fields, G., Sutton, L., 1996. GEM-2: A New Multifrequency Electromagnetic Sensor. Journal of Environmental and. Engineering Geophysics, 1, $129-137$.

\section{Figure captions}

Figure 1: In-field data acquisition in HCP geometry for the GEM-2 (Geophex Ltd.) on a Neolithic magoules (credit: Meropi Manataki). Instrument is hold at a height of $0.3 \mathrm{~m}$ in a hand operating acquisition mode.

Figure 2: Opposites of the responses, expressed by $\mathrm{Hs} / \mathrm{Hp}$ ratio, (a) versus conductivity for $\kappa_{p h}$ $=50.10^{-5}$ S.I., and (b) versus in-phase susceptibility for $\sigma=50 \Omega . m$ (the quadrature susceptibility being $6 \%$ of the in-phase), at $5 \mathrm{kHz}$ and $40 \mathrm{kHz}$ for the GEM-2 instrument when held at $0.3 \mathrm{~m}$ height above ground surface.

Figure 3: In-phase (a) and quadrature response (b) as functions of the height above ground surface, GEM-2, for $\kappa_{p h}=5010^{-5}$ S.I., and $f=5010 \mathrm{~Hz}$. 
475 Figure 4: Quadrature response as functions of the quadrature out-of-phase part of the magnetic susceptibility for both 100 and $500 \Omega . m$ and $f=5010 \mathrm{~Hz}$

477 Figure 5 : (a) Apparent magnetic susceptibility and (b) apparent magnetic viscosity variations 478 with increasing the thickness of the first layer for $5010 \mathrm{~Hz}$ and $13370 \mathrm{~Hz}$ frequencies.

479 Figure 6: Value of the apparent magnetic viscosity obtained with the proposed procedure as a 480 function of electrical conductivity and magnetic viscosity a) $f=5010 \mathrm{~Hz}$ and b) $f=13370 \mathrm{~Hz}$.

481 Figure 7: Result of the processing of synthetic data, a) simulated model, b) apparent electrical 482 conductivity, c) apparent magnetic susceptibility for $5010 \mathrm{~Hz}$, d) apparent magnetic 483 susceptibility for $13370 \mathrm{~Hz}$, e) apparent magnetic viscosity for $5010 \mathrm{~Hz}$, f) apparent magnetic viscosity for $13370 \mathrm{~Hz}$.

Figure 8: Raw data for the site of Karatzantakli (Grece): a) In-phase measurement in ppm for $5010 \mathrm{~Hz}$, b) In-phase measurement for $13370 \mathrm{~Hz}$, c) Quadrature out-of-phase measurement for $5010 \mathrm{~Hz}$, d) Quadrature out-of-phase measurement for $13370 \mathrm{~Hz}$.

Figure 9: Processed data for the site of Karatzantakli (Greece): a) apparent electrical conductivity, b) apparent magnetic susceptibility for $5010 \mathrm{~Hz}$, c) apparent magnetic susceptibility for $13370 \mathrm{~Hz}$, d) apparent magnetic viscosity for $5010 \mathrm{~Hz}$, e) apparent magnetic viscosity for $13370 \mathrm{~Hz}$.

492 Figure 10: Raw data for the site of Almiriotiki (Greece): a) In-phase measurement in ppm for $5010 \mathrm{~Hz}$, b) In-phase measurement for $13370 \mathrm{~Hz}$, c) Quadrature out-of-phase measurement 494 for $5010 \mathrm{~Hz}$, d) Quadrature out-of-phase measurement for $13370 \mathrm{~Hz}$. Figure 11: Processed data for the Neolithic site of Almiriotiki (Greece): a) apparent electrical conductivity, b) apparent magnetic susceptibility for $5010 \mathrm{~Hz}$, c) apparent magnetic 
497 susceptibility for $13370 \mathrm{~Hz}$, d) apparent magnetic viscosity for $5010 \mathrm{~Hz}$, e) apparent magnetic

498 viscosity for $13370 \mathrm{~Hz}$.

499

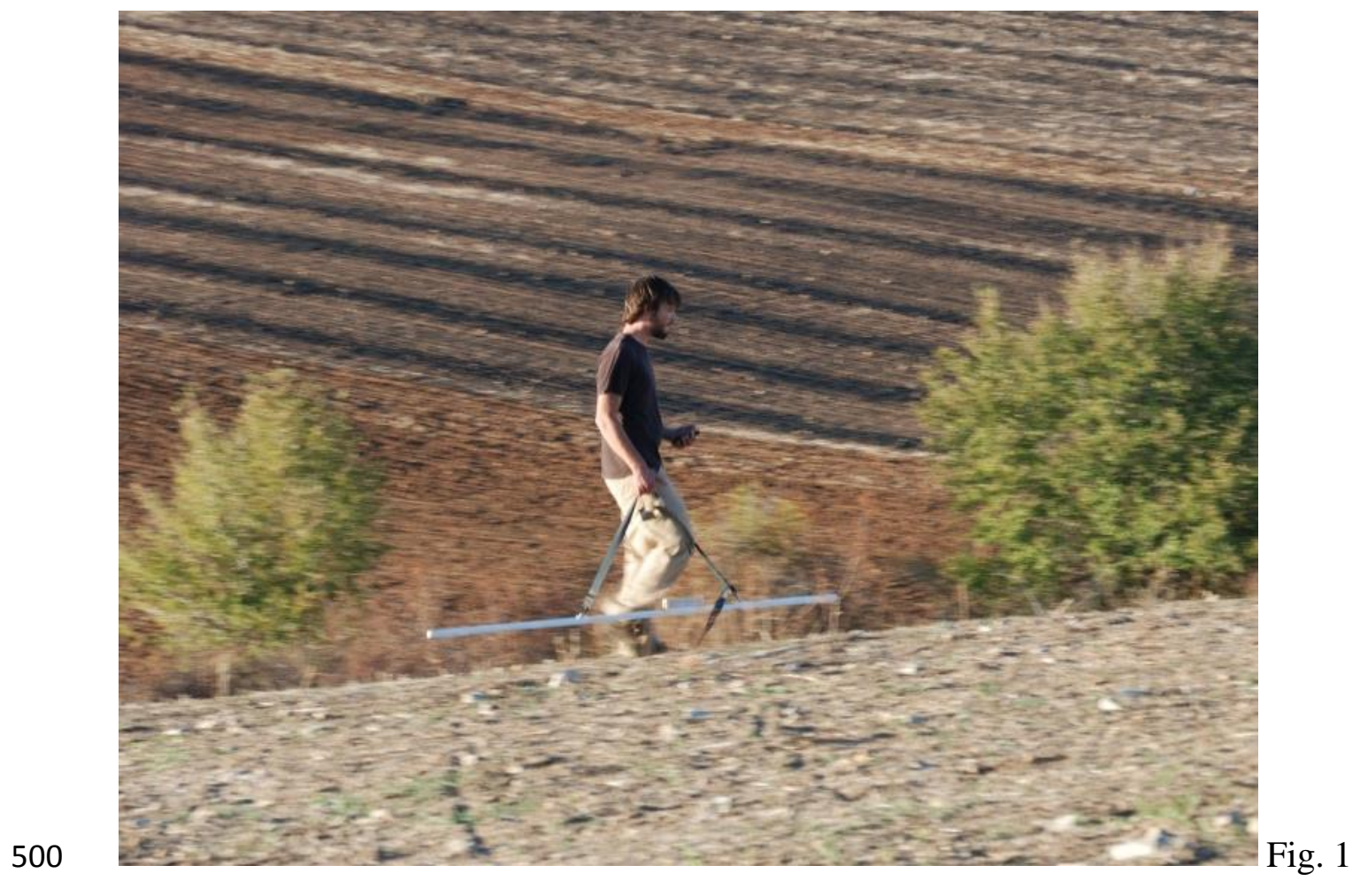

501 


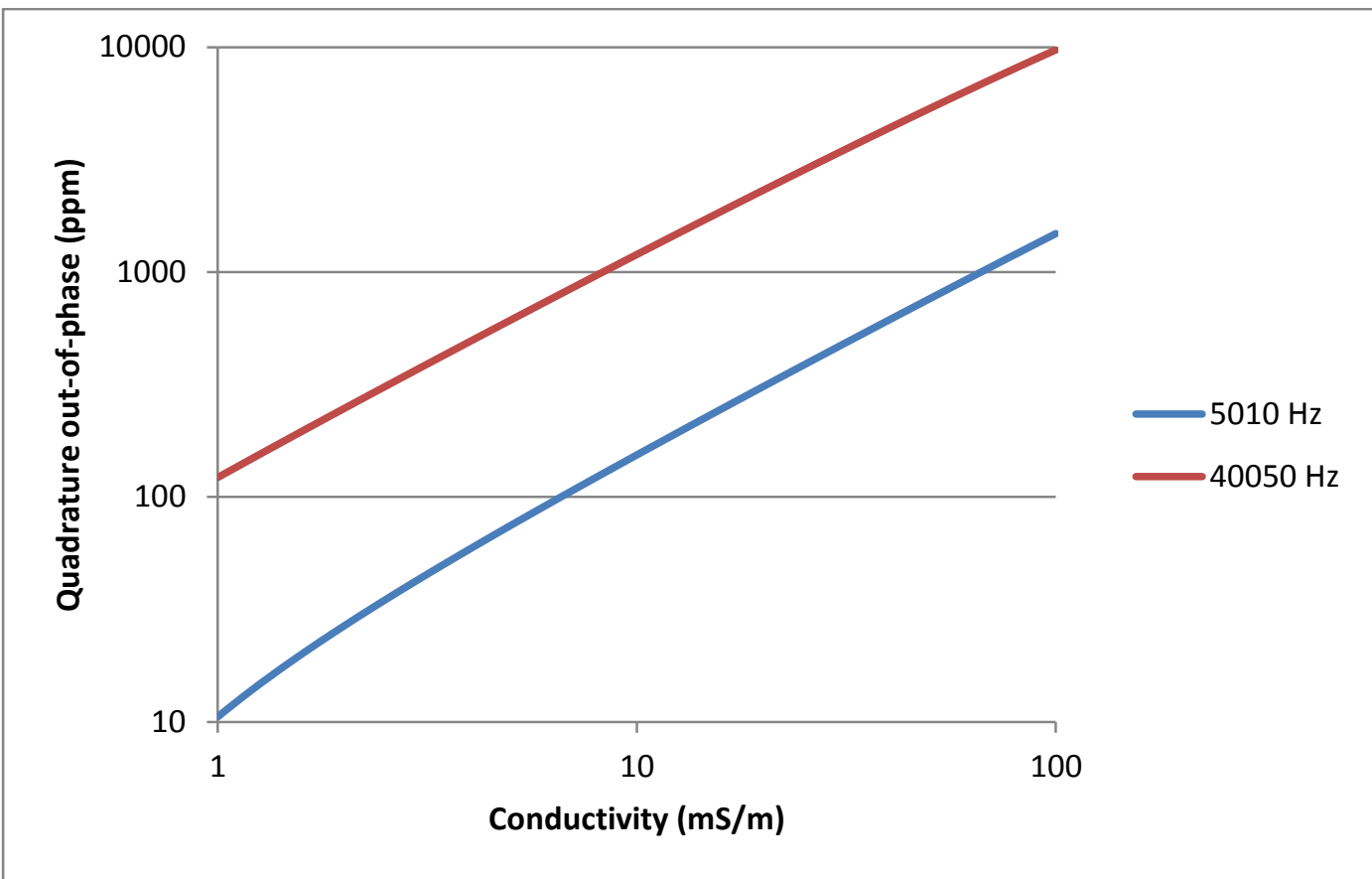

Fig. 2a

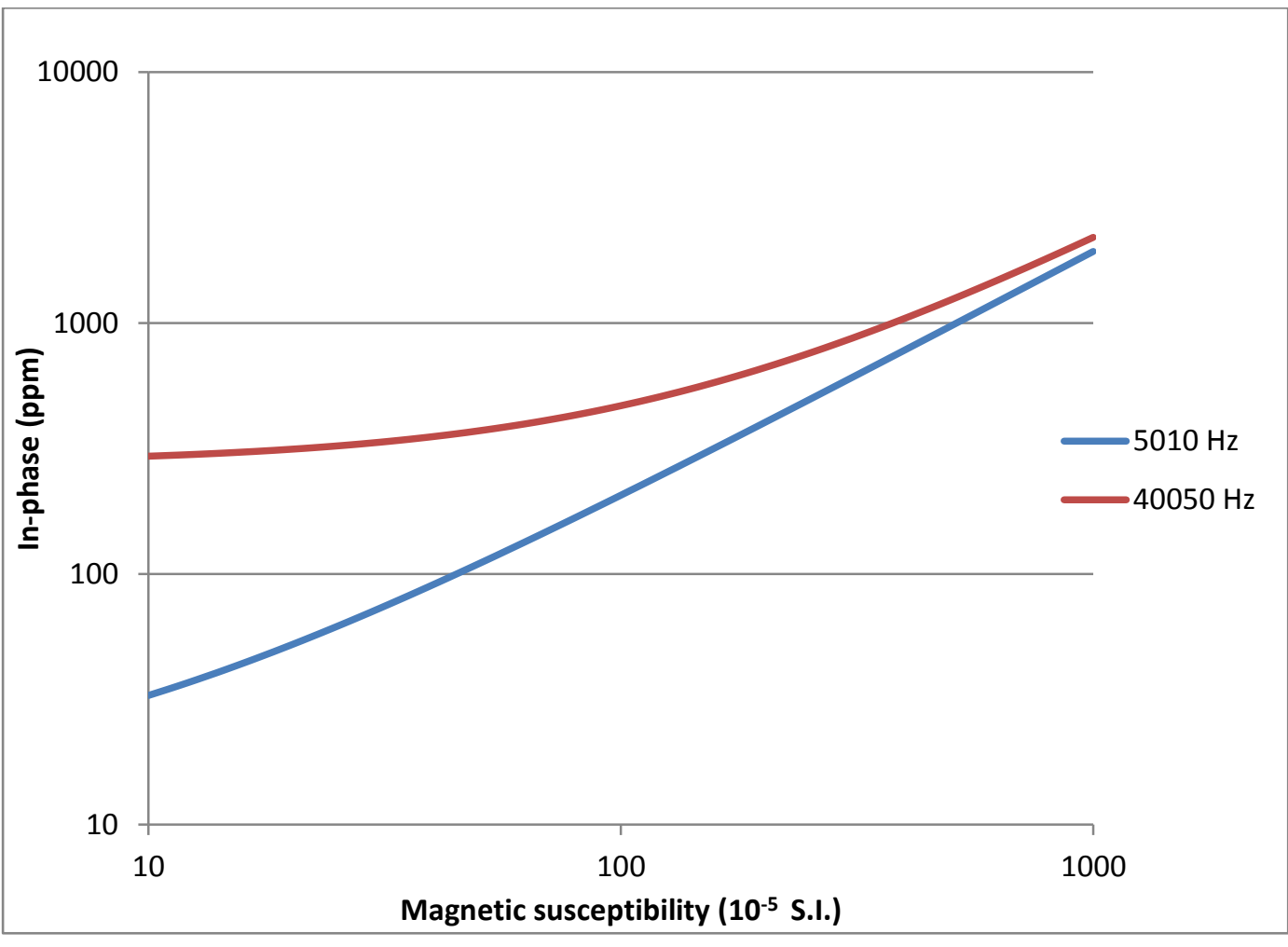

Fig. $2 b$

505 


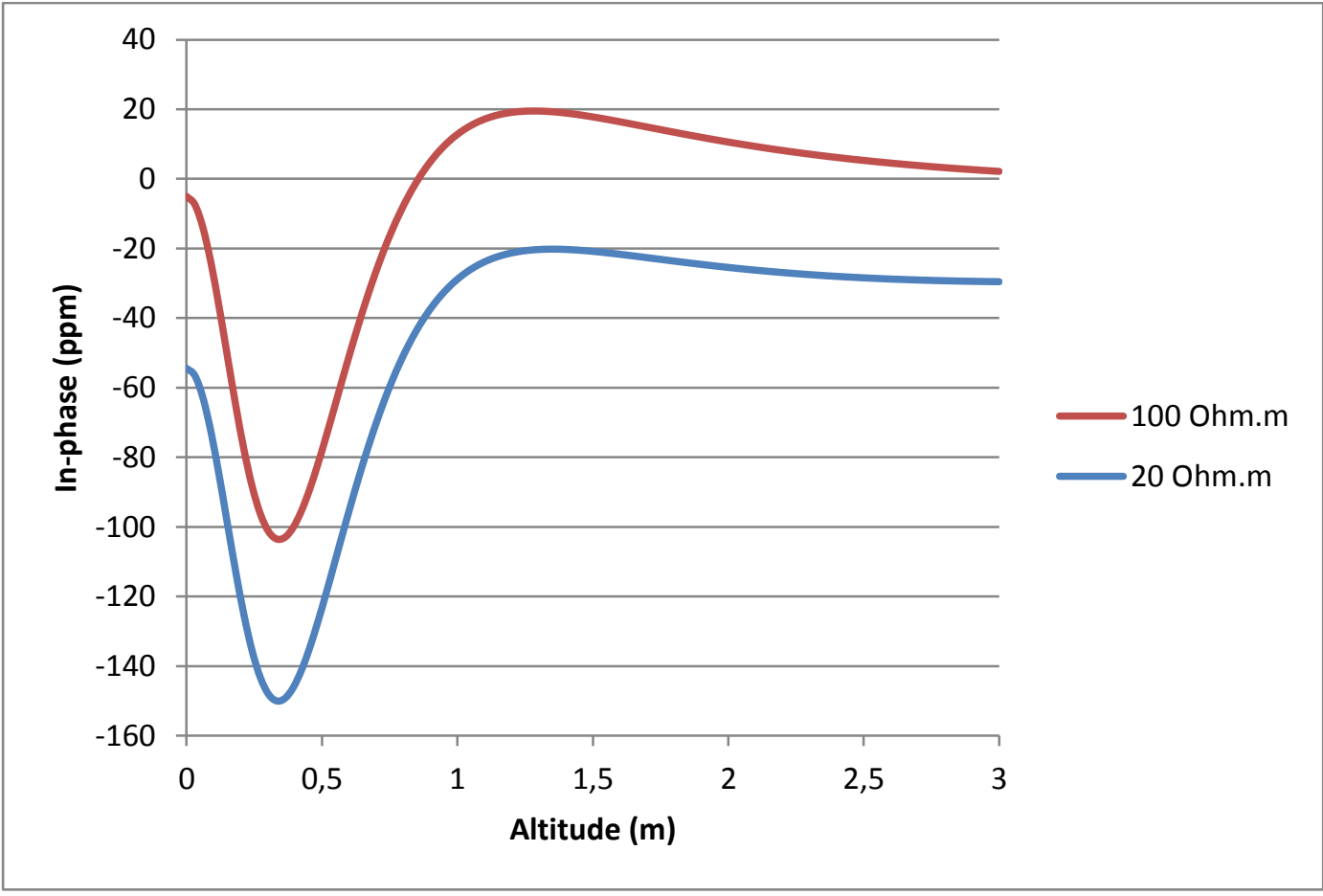

Fig. 3a

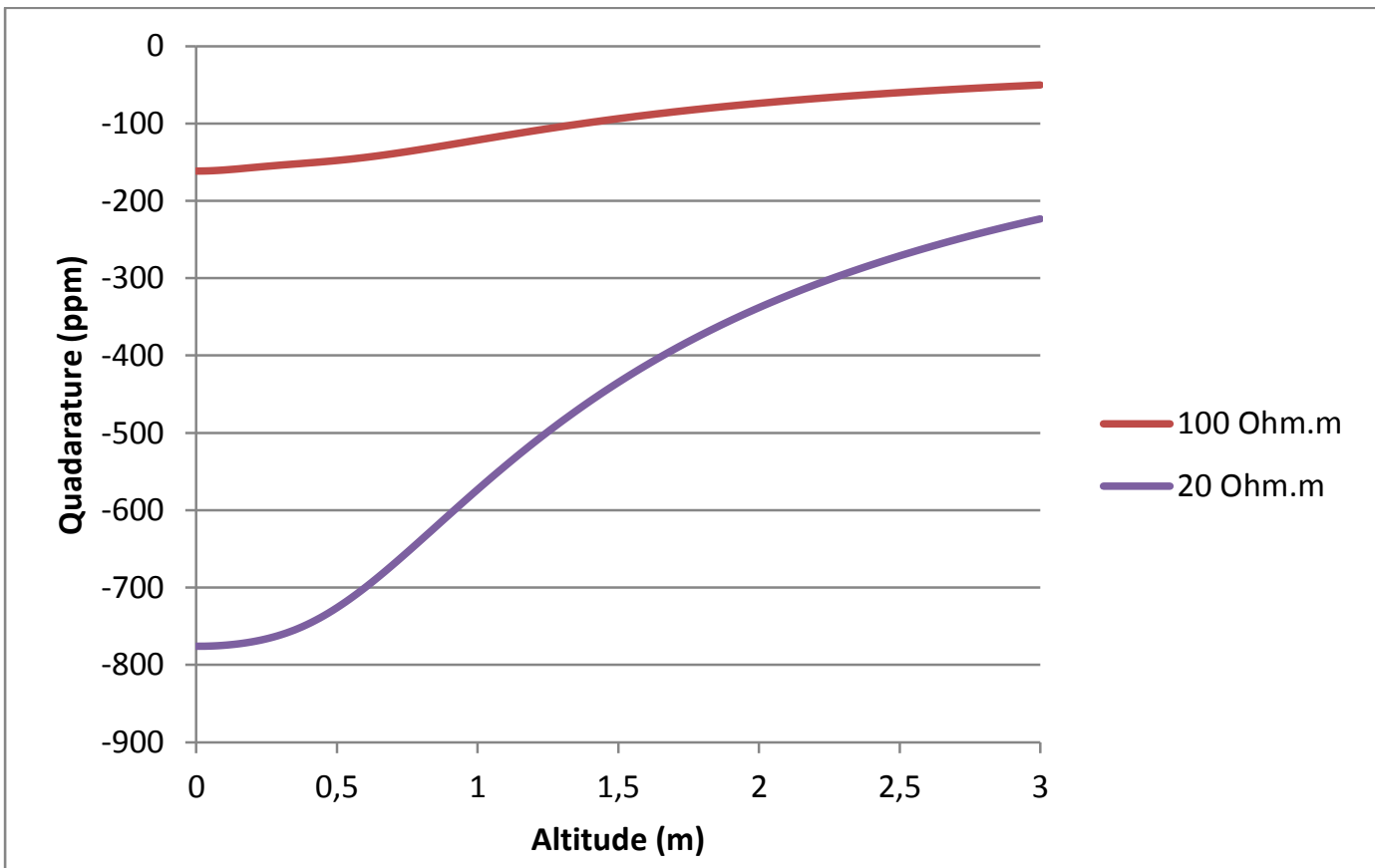

Fig. 3b 


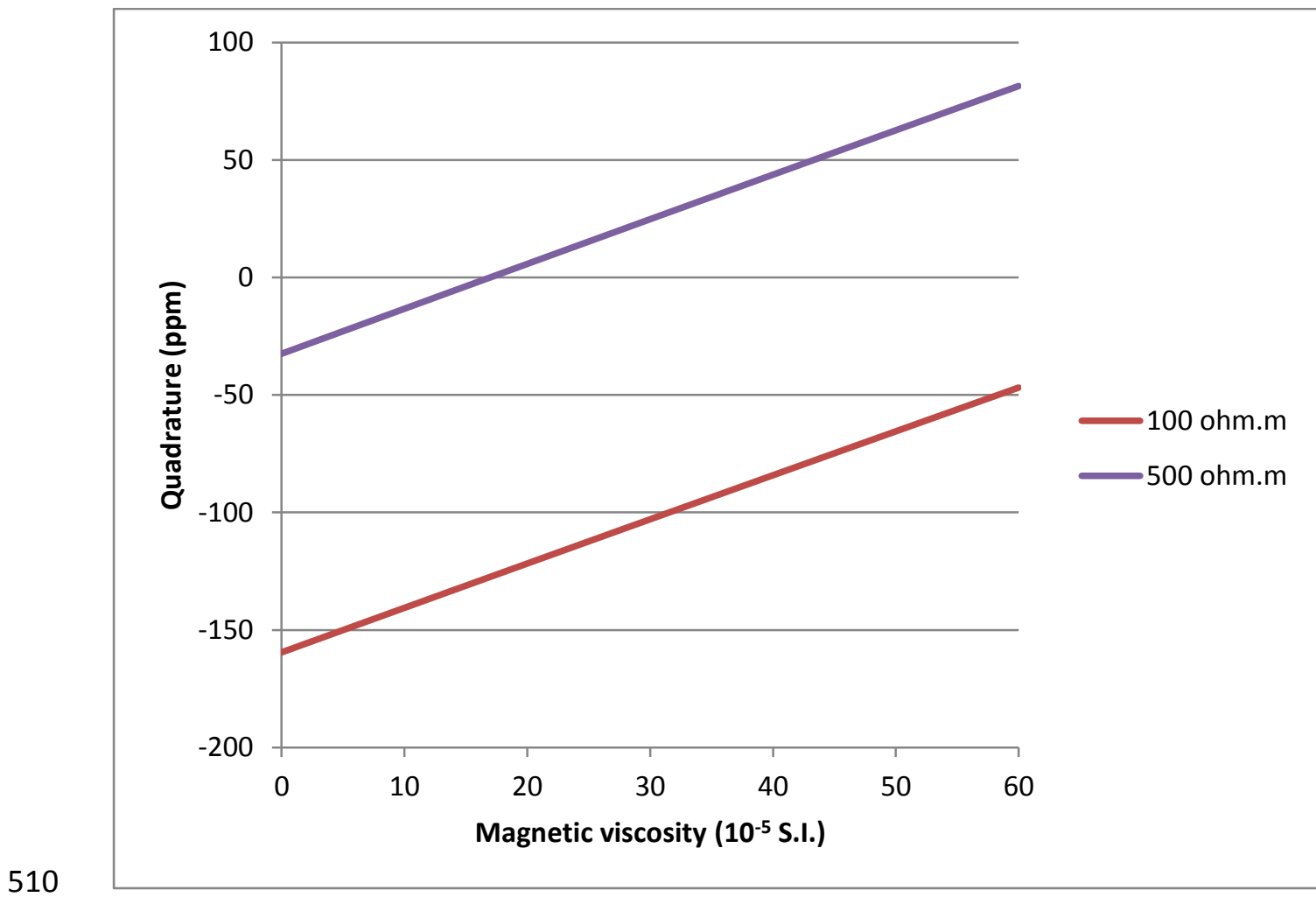

Fig. 4

511

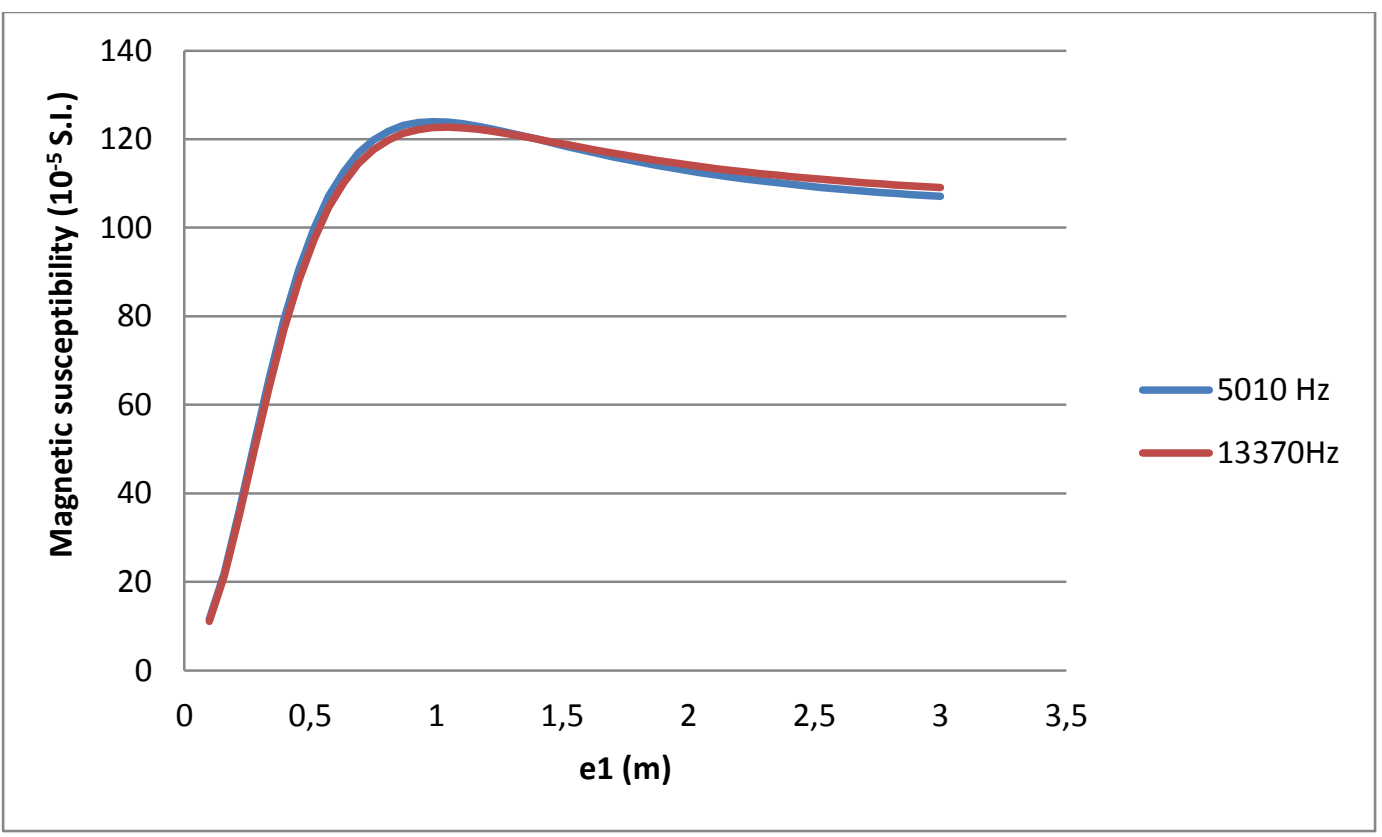

Fig. 5a 


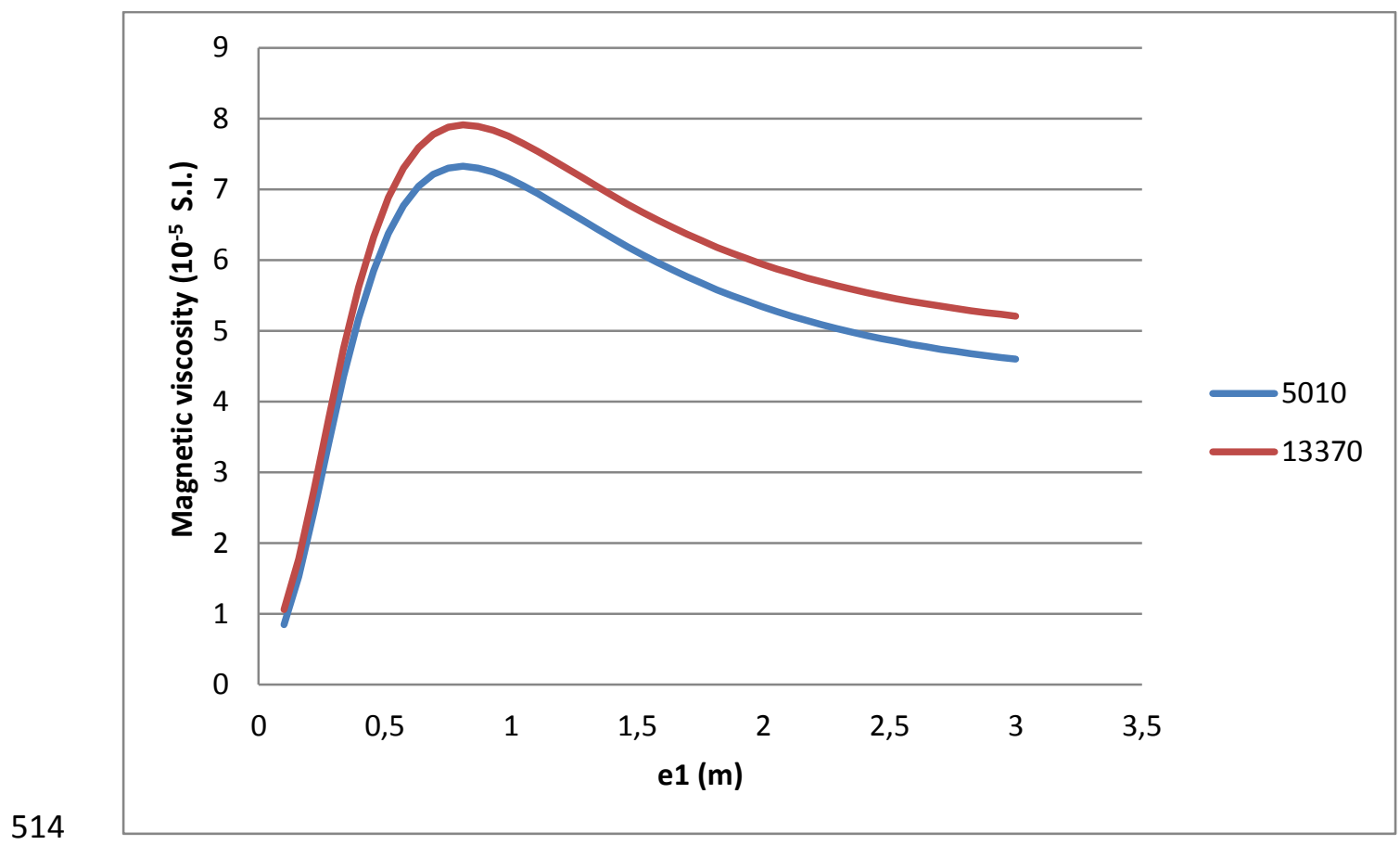

Fig. $5 b$

515

516

517 


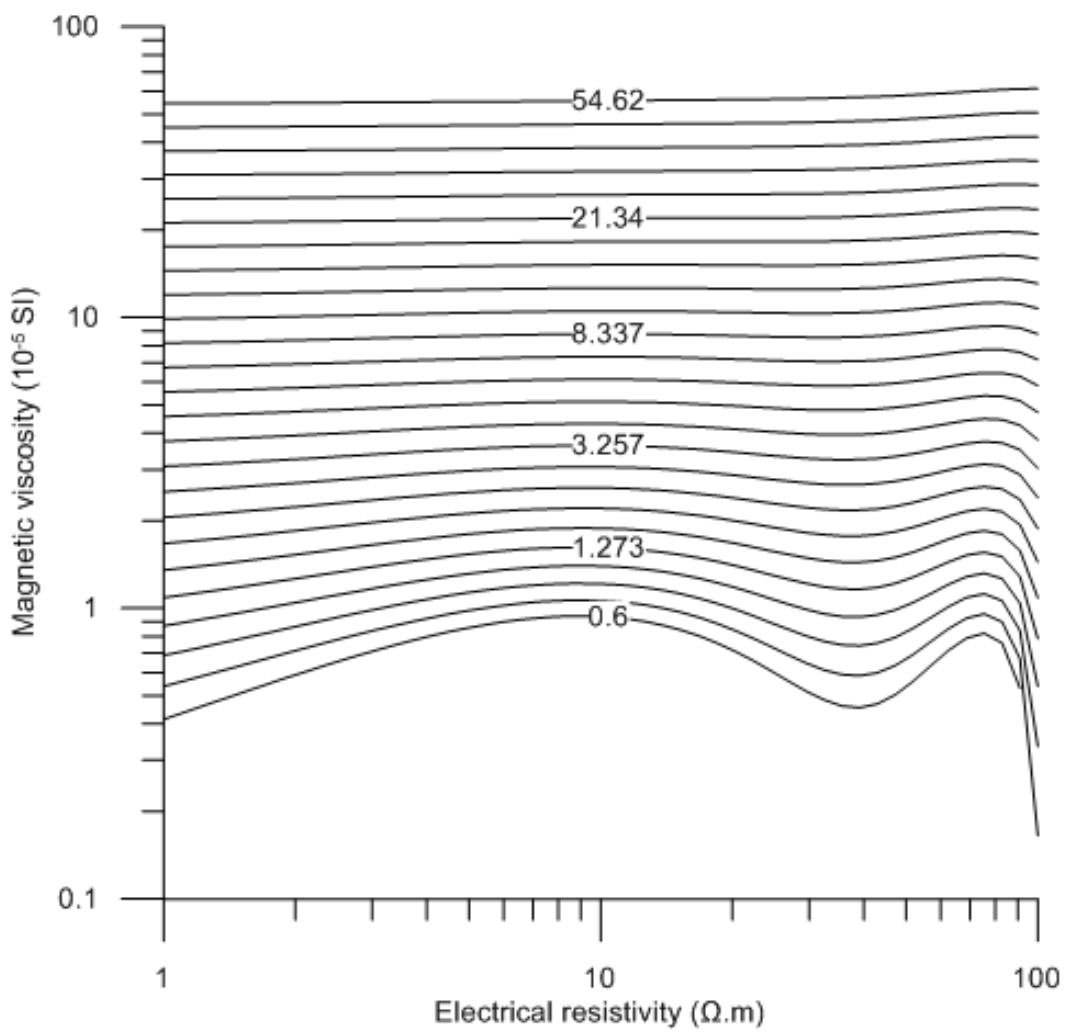

a. Value of the apparent magnetic viscosity obtained with the proposed procedure as function of electrical conductivity and magnetic viscosity $f=5010 \mathrm{~Hz}$

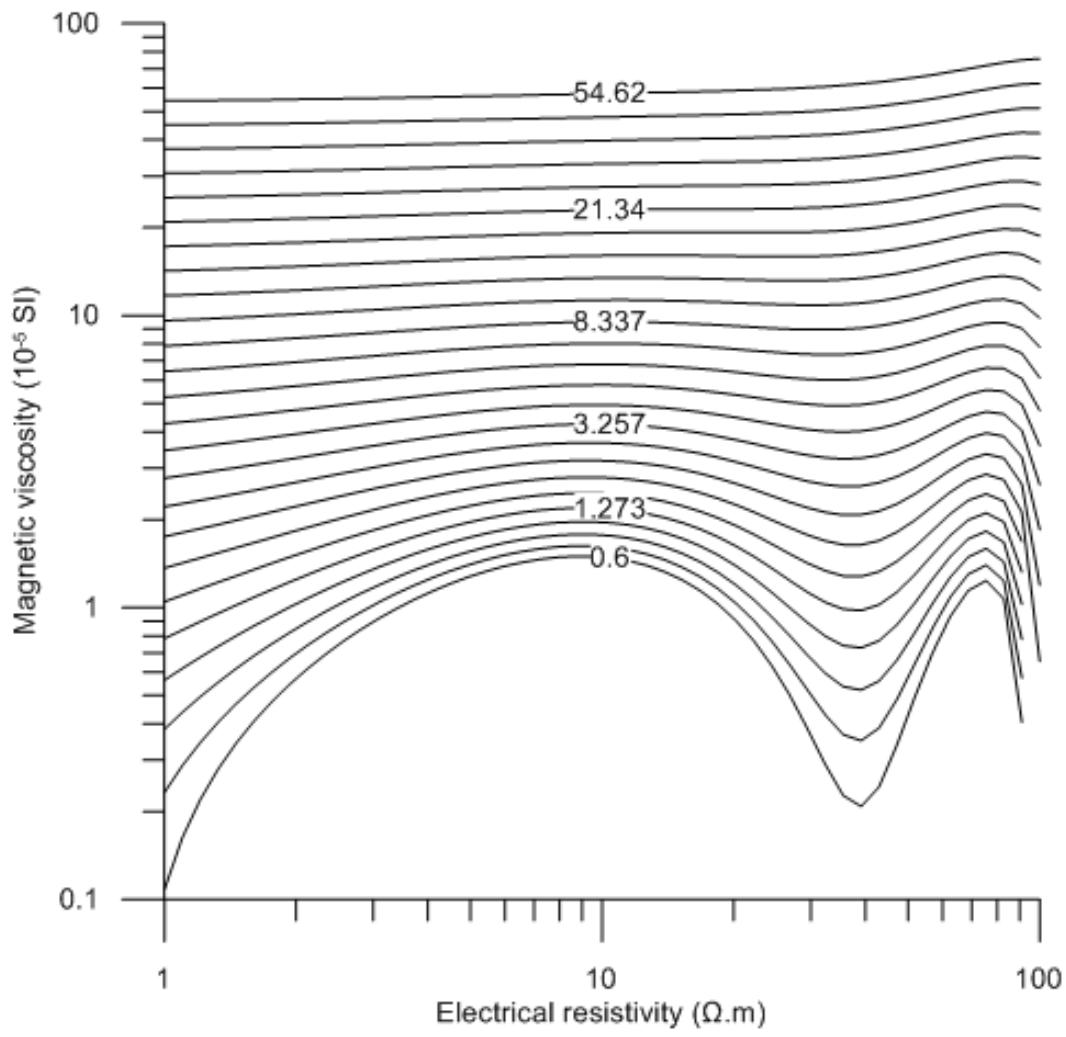

b. Value of the apparent magnetic viscosity obtained with the proposed procedure as function of electrical conductivity and magnetic viscosity at $f=13370 \mathrm{~Hz}$ 

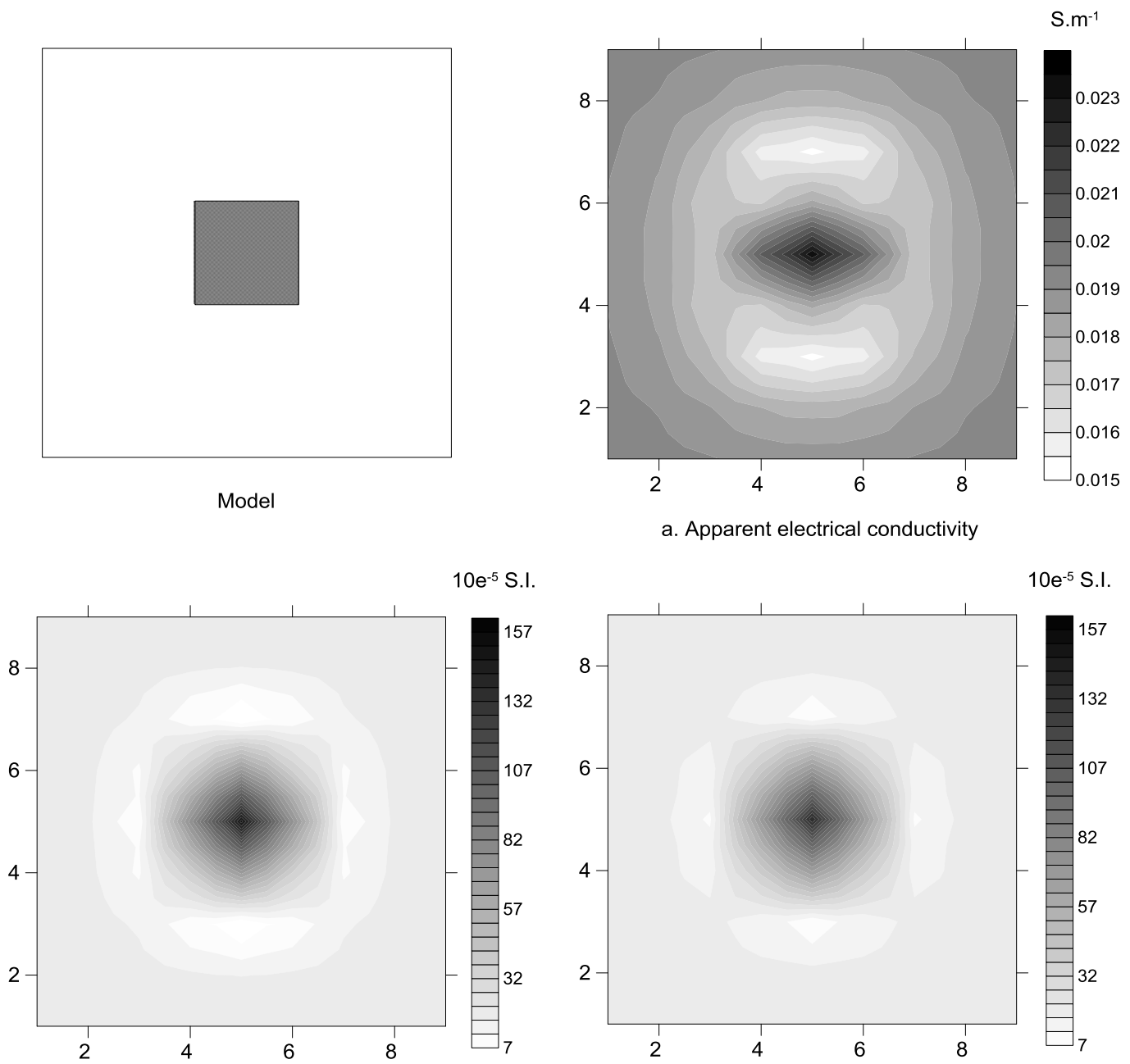

$10 \mathrm{e}^{-5}$ S.I.

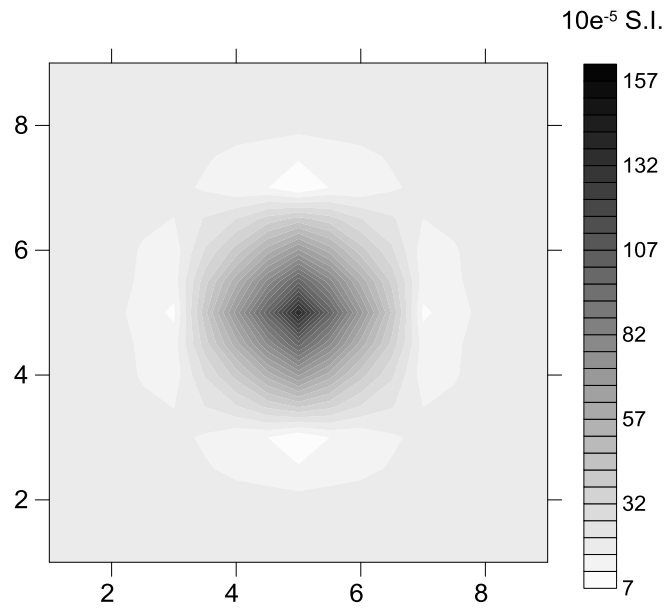

b. Apparent magnetic susceptibility $(5010 \mathrm{~Hz})$

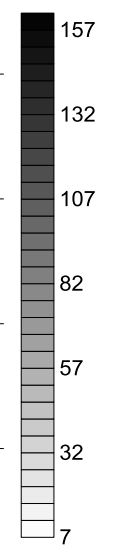

c. Apparent magnetic susceptibility $(13370 \mathrm{~Hz})$
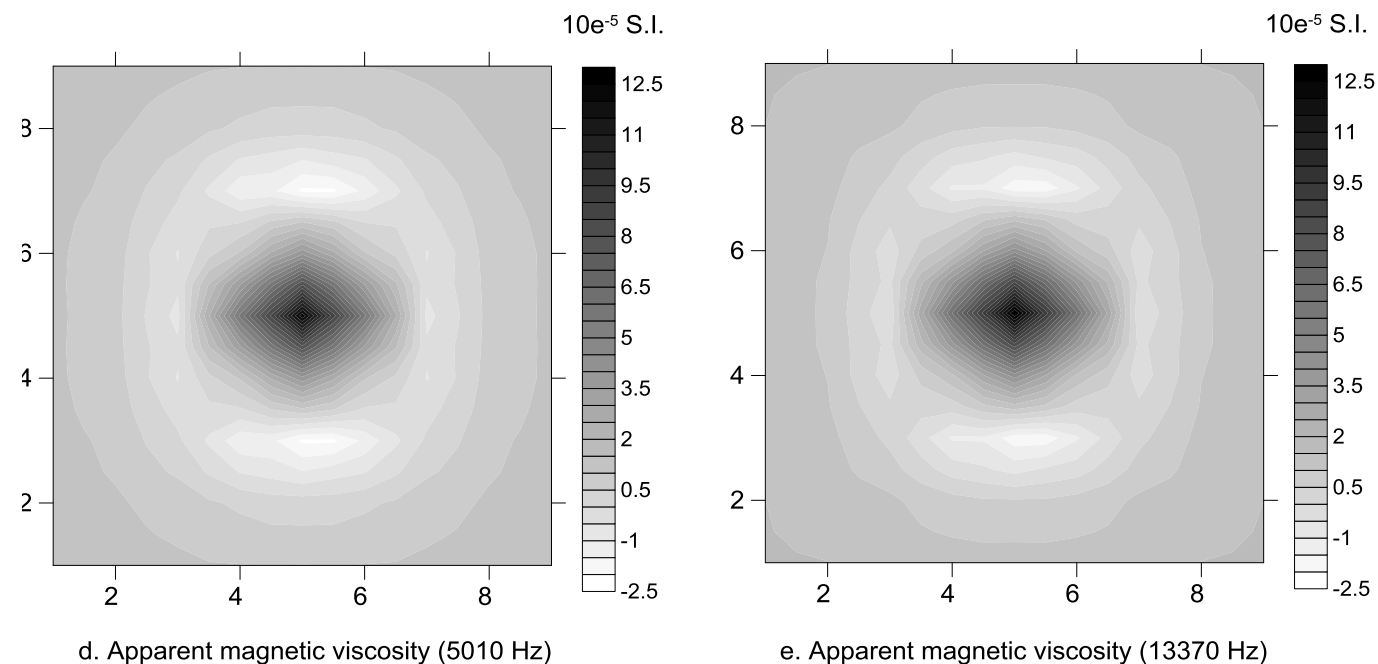
a. Raw in-phase component measurement $(5010 \mathrm{hZ})$

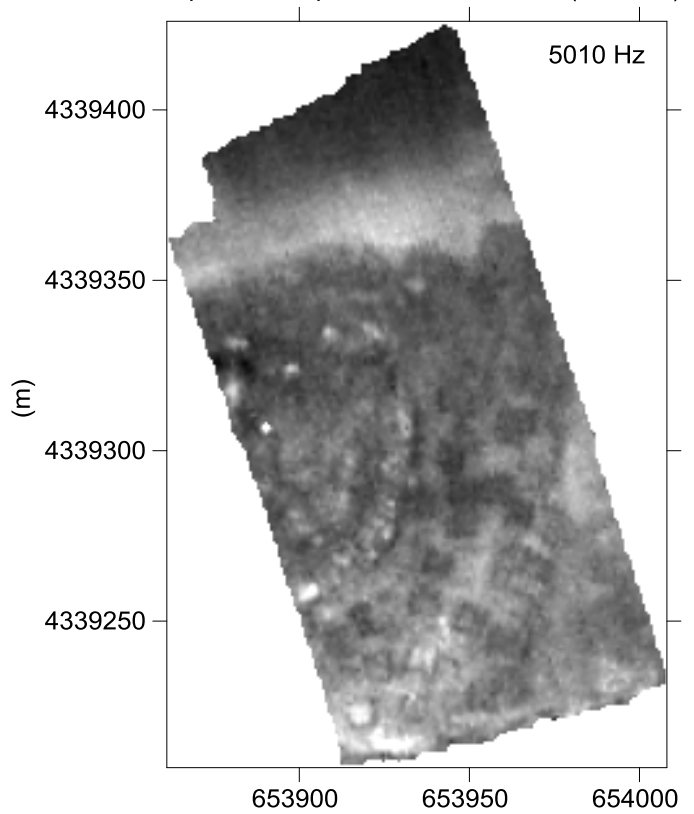

(m)

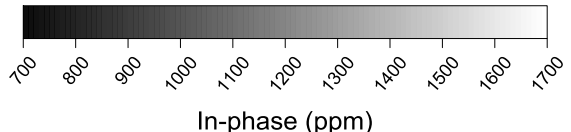

c. Raw quadrature component measurement $(5010 \mathrm{~Hz})$

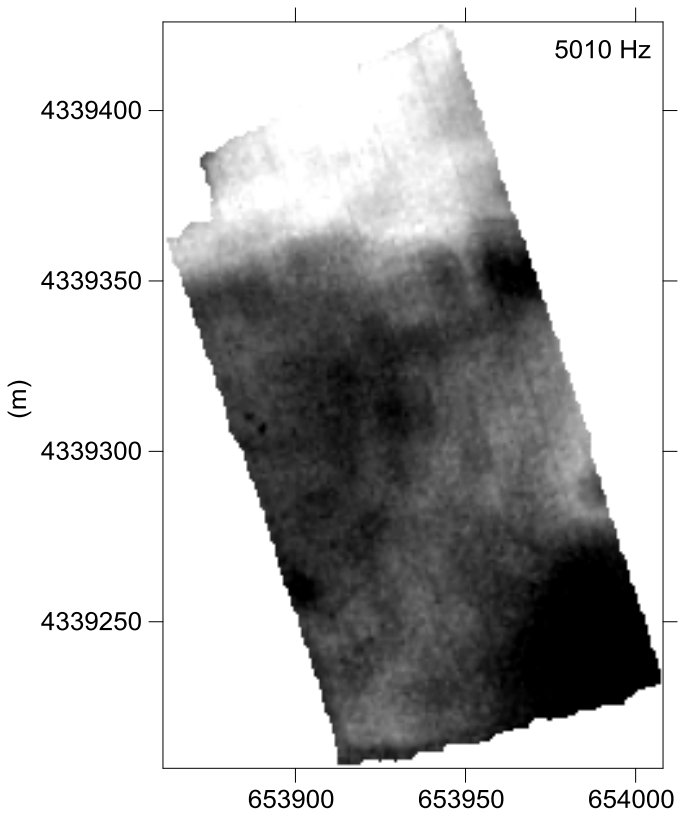

(m)

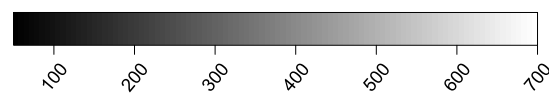

b. Raw in-phase component measurement (13370 hZ)

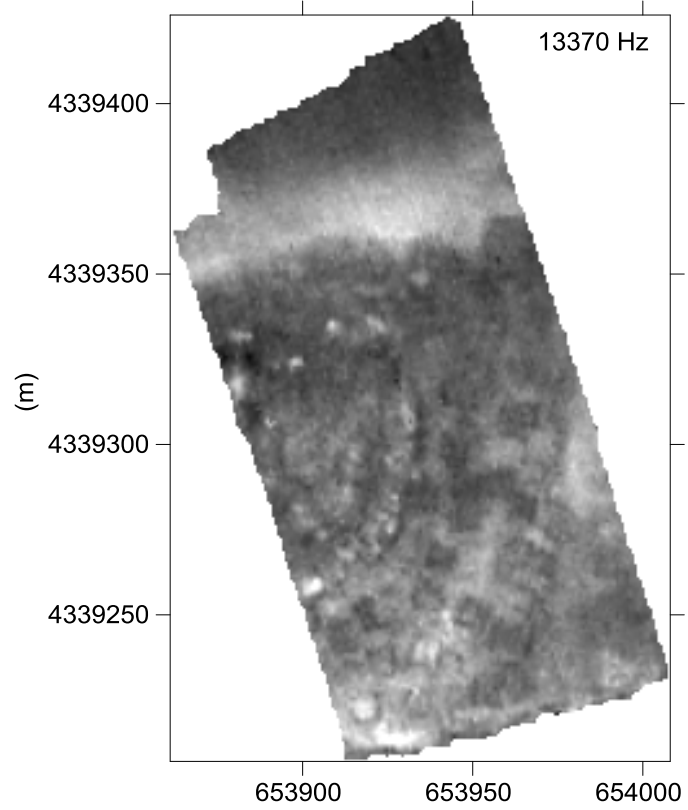

(m)

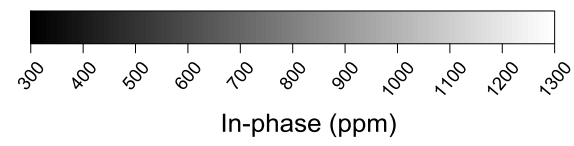

d. Raw quadrature component measurement $(13370 \mathrm{~Hz})$

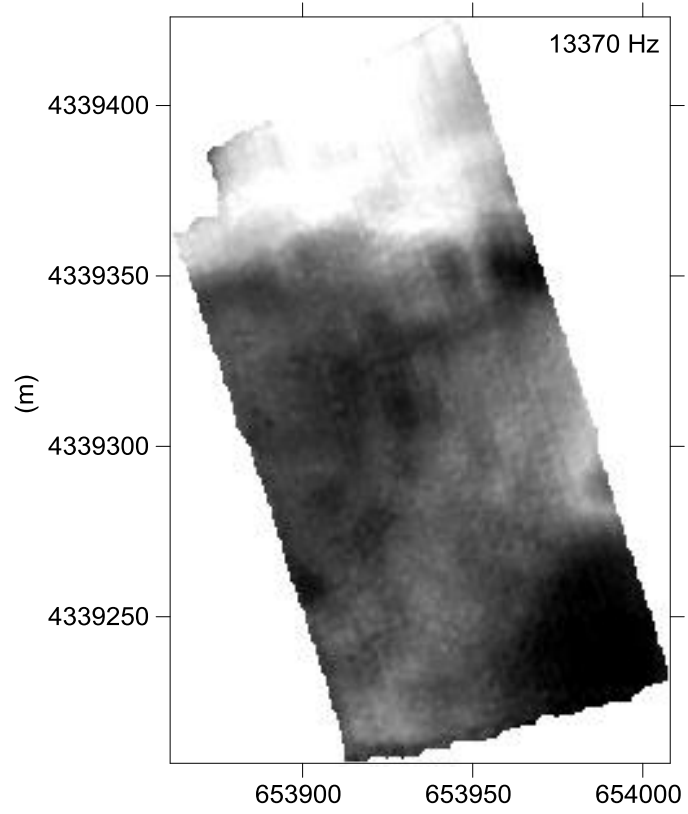

(m)

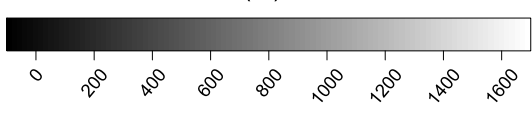

Quadrature (ppm)

$525 \quad$ Fig. 8 


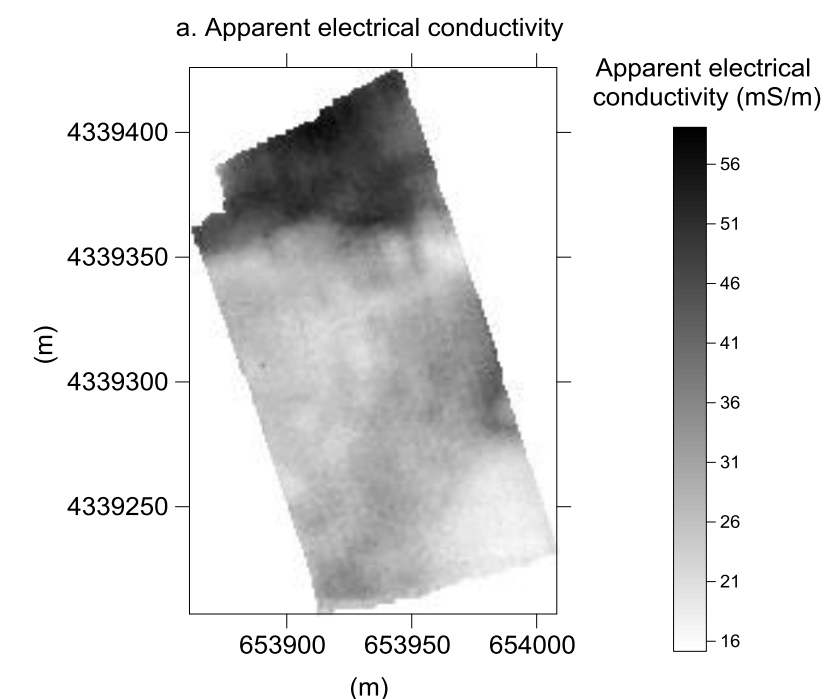

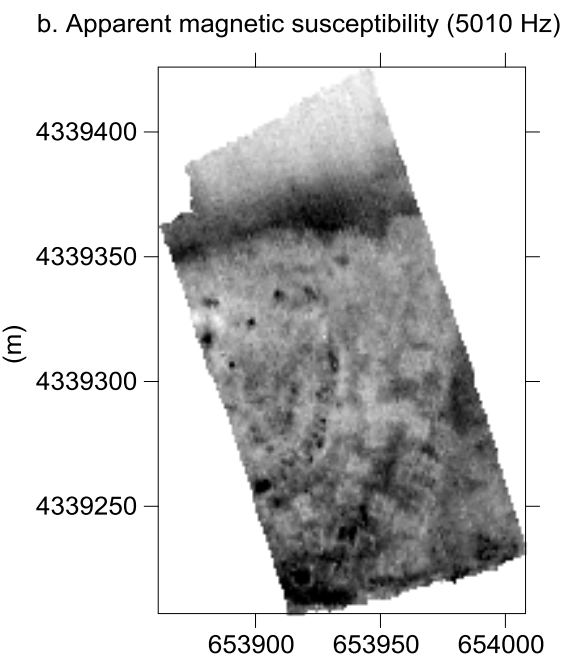

$(\mathrm{m})$

d. Apparent magnetic viscosity $(5010 \mathrm{~Hz})$

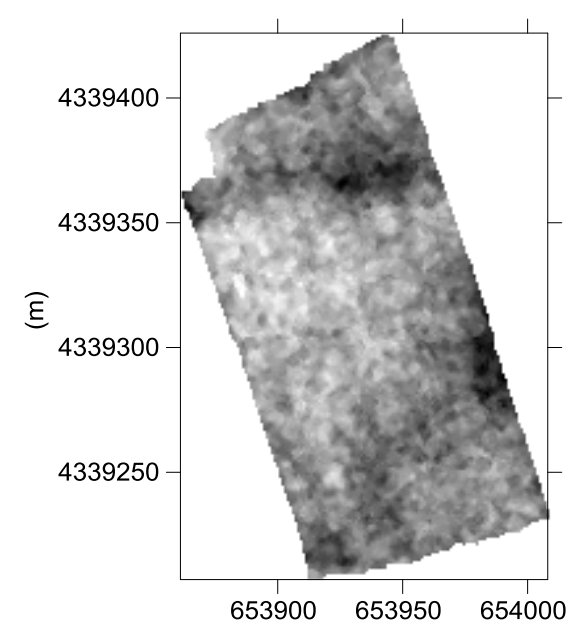

(m)

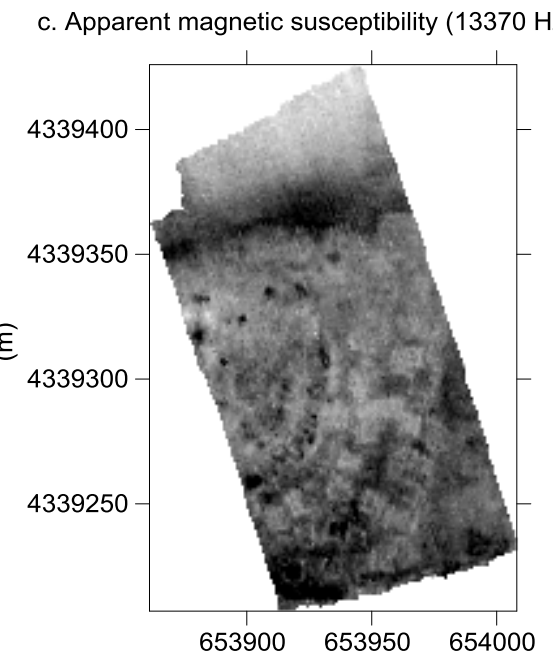

(m)
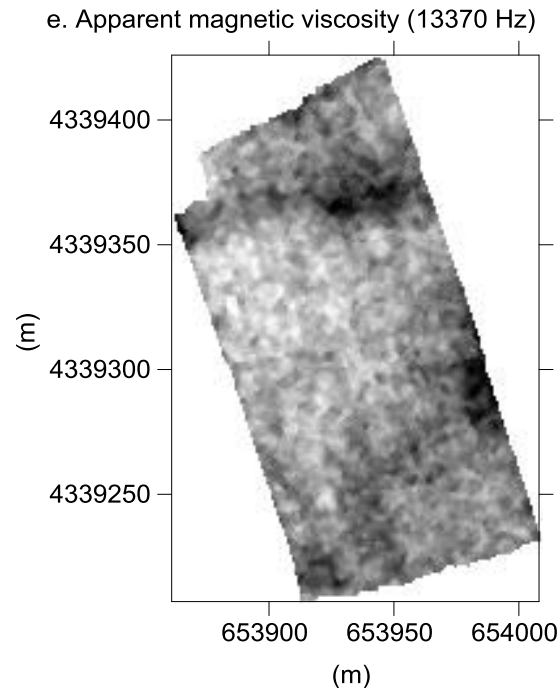

Apparent magnetic viscosity $\left(10^{-5} \mathrm{~S} . \mathrm{I}\right.$.

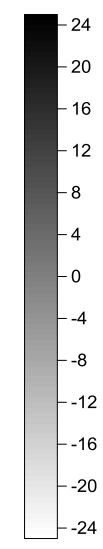

(m)

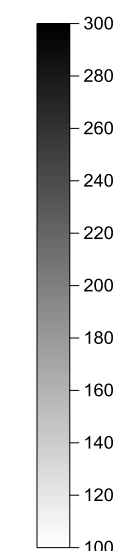

Apparent magnetic susceptibility $\left(10^{-5}\right.$ S.I.)

$527 \quad$ Fig. 9 
a. Raw in-phase component measurement $(5010 \mathrm{hZ})$
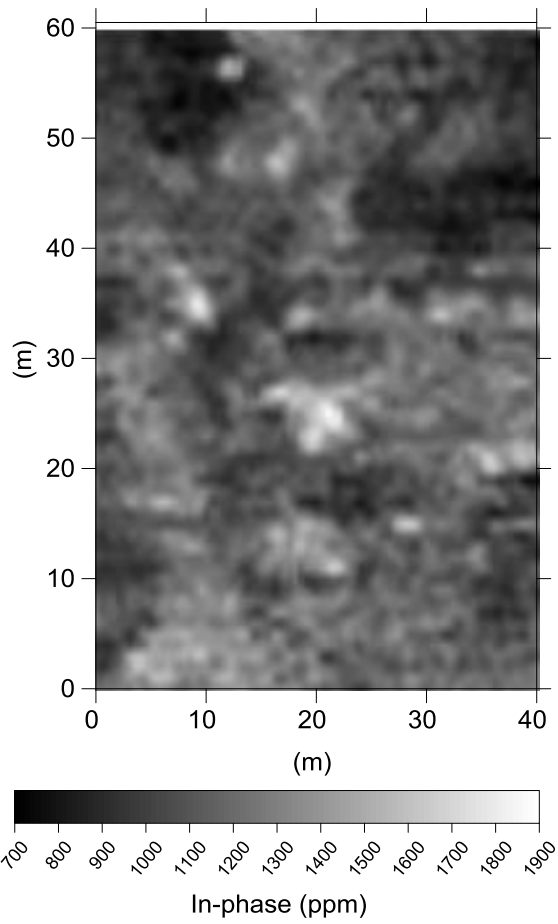

c. Raw quadrature component measurement $(5010 \mathrm{hZ})$
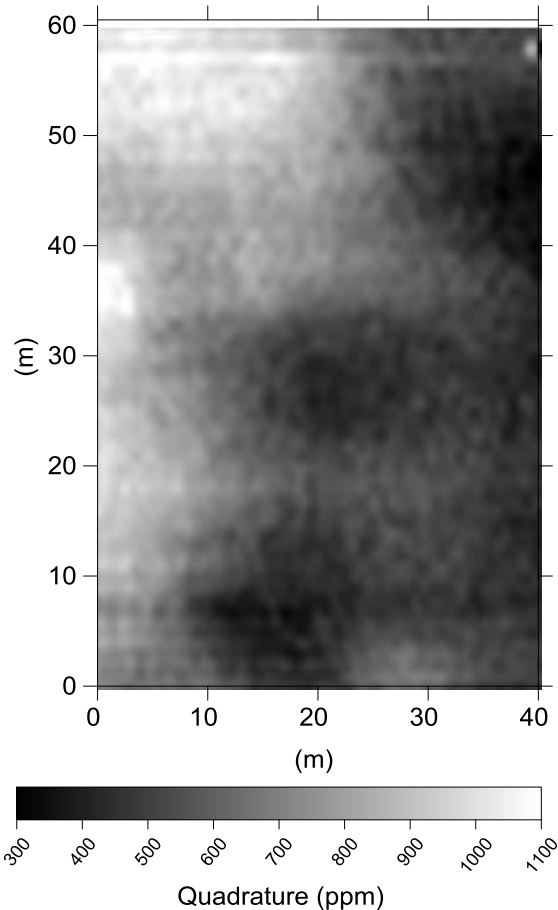

b. Raw in-phase component measurement (13370 hZ)
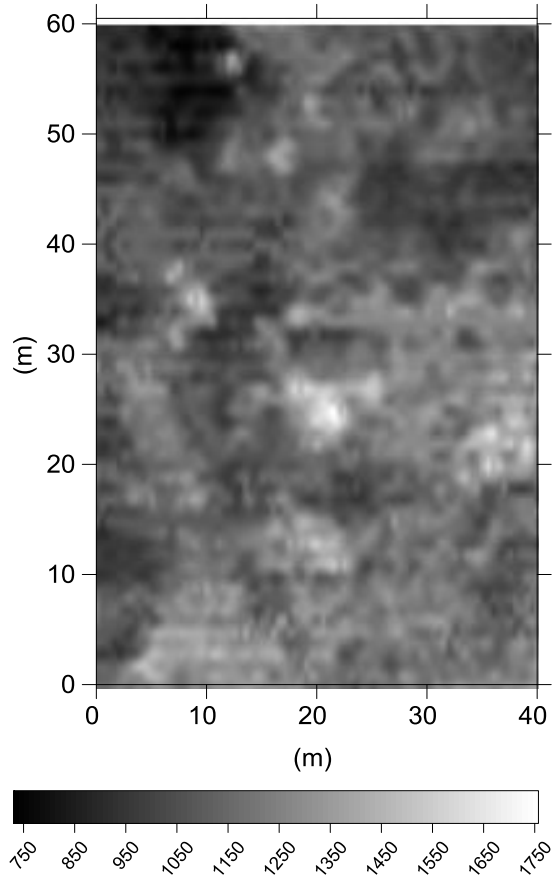

In-phase (ppm)

d. Raw quadrature component measurement (13370 hZ)

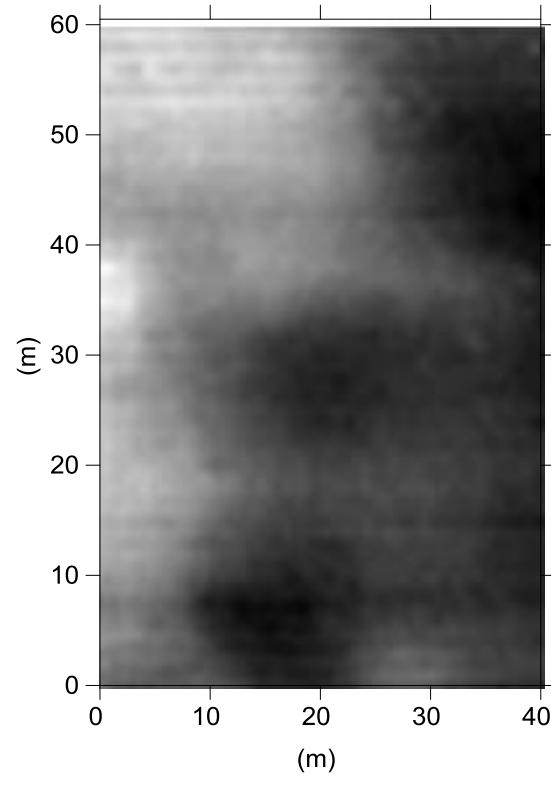

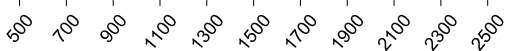
Quadrature (ppm)

529 Fig. 10 


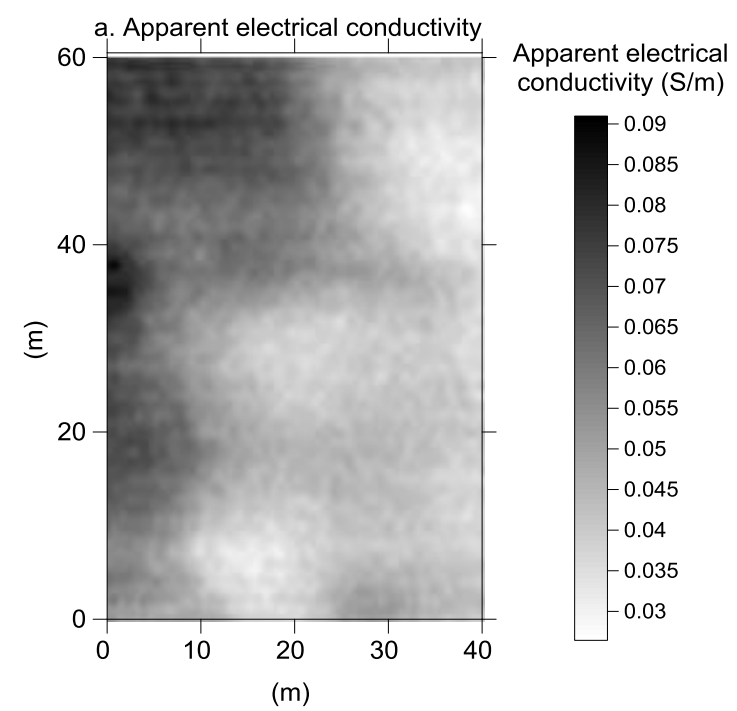

b. Apparent magnetic susceptibility $(5010 \mathrm{hZ})$

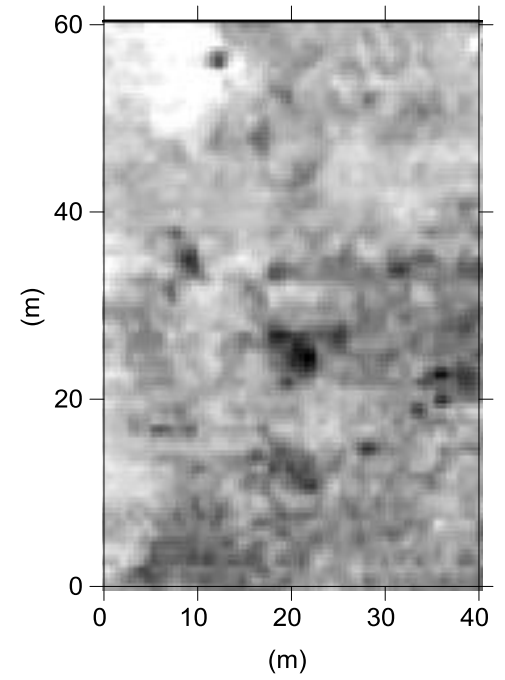

d. Apparent magnetic viscosity (5010 hZ)

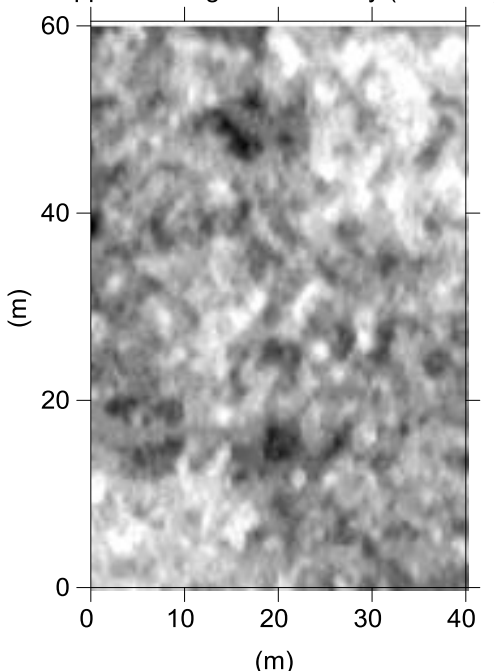

(m)

c. Apparent magnetic susceptibility (13370 hZ)

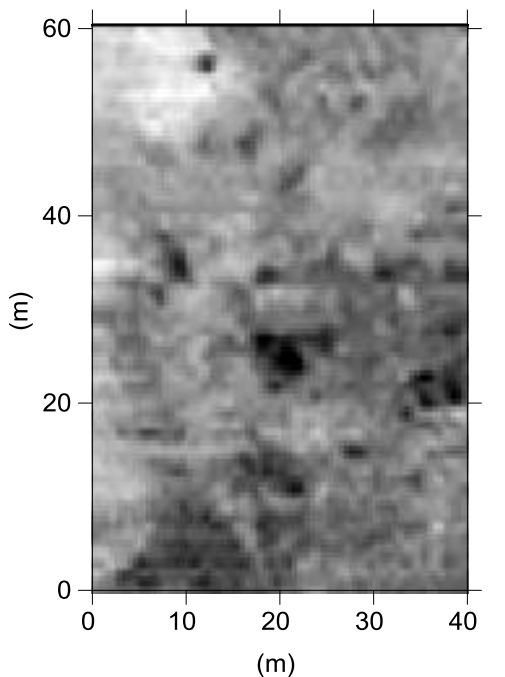

e. Apparent magnetic viscosity $(13370 \mathrm{hZ})$

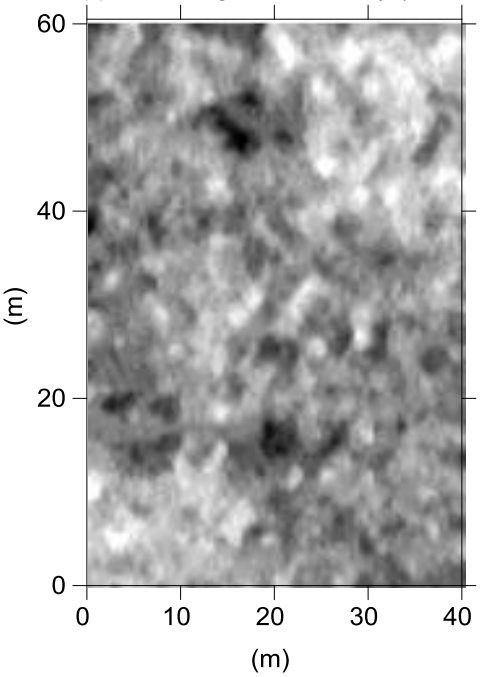

(m)
Apparent magnetic susceptibility $\left(10^{-5}\right.$ S.I.)
Apparent magnetic viscosity $\left(10^{-5}\right.$ S.I.)

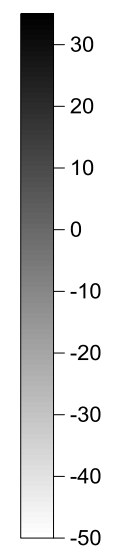

Fig. 11 